\title{
EVOLUTION OF THE GLOBAL TEMPERATURE STRUCTURE OF THE SOLAR CORONA DURING THE MINIMUM BETWEEN SOLAR CYCLES 23 AND 24
}

\author{
Federico A. Nuevo ${ }^{1}$, Zhenguang Huang $^{2}$, Richard Frazin $^{2}$, Ward B. Manchester iv ${ }^{2}$, \\ Meng Jin ${ }^{2}$, AND Alberto M. VÁsQueZ ${ }^{1}$ \\ ${ }^{1}$ Instituto de Astronomía y Física del Espacio (CONICET-UBA) and FCEN (UBA), CC 67-Suc 28, Ciudad de Buenos Aires, Argentina \\ ${ }^{2}$ Department of Atmospheric, Oceanic and Space Sciences, University of Michigan, Ann Arbor, MI 48109, USA \\ Received 2013 March 8; accepted 2013 May 3; published 2013 July 19
}

\begin{abstract}
The combination of differential emission measure tomography with extrapolation of the photospheric magnetic field allows determination of the electron density and electron temperature along individual magnetic field lines. This is especially useful in quiet-Sun (QS) plasmas where individual loops cannot otherwise be identified. In Paper I, this approach was applied to study QS plasmas during Carrington rotation (CR) 2077 at the minimum between solar cycles (SCs) 23 and 24. In that work, two types of QS coronal loops were identified: "up" loops in which the temperature increases with height, and "down" loops in which the temperature decreases with height. While the first ones were expected, the latter ones were a surprise and, furthermore, were found to be ubiquitous in the low-latitude corona. In the present work, we extend the analysis to $11 \mathrm{CRs}$ around the last solar minimum. We found that the "down" population, always located at low latitudes, was maximum at the time when the sunspot number was minimum, and the number of down loops systematically increased during the declining phase of SC-23 and diminished during the rising phase of SC-24. "Down" loops are found to have systematically larger values of $\beta$ than do "up" loops. These discoveries are interpreted in terms of excitation of Alfvén waves in the photosphere, and mode conversion and damping in the low corona.
\end{abstract}

Key words: Sun: corona - Sun: evolution - Sun: magnetic topology - Sun: UV radiation - sunspots

Online-only material: color figures

\section{INTRODUCTION}

Observational evidence of coronal heating, in the form of temperature diagnostics of the Sun's corona, is needed to improve our understanding of the solar atmosphere. The combination of differential emission measure tomography (DEMT; based on time series of EUV images) with magnetic field models of the solar corona can provide global temperature constraints for the lower corona (below $1.25 R_{\odot}$ ). This approach, named the Michigan Loop Diagnostic Technique (MLDT), is based on tomographic reconstructions and hence it is a reliable description of the stable regions of the corona, in particular regions of the quiet (diffuse) corona for which individual loops cannot be individually distinguished in the EUV or X-ray images. MLDT was first used to study Carrington rotation (CR) 2077 during the minimum between solar cycles (SCs) 23 and 24 (Huang et al. 2012, hereafter Paper I). By tracing the tomographic temperature variation along many quiet-Sun (QS) loops, it was found that, for a large number of them, the temperature decreases with height. These loops were called "down" loops. Furthermore, it was found that down loops were characteristic of the equatorial region, being mainly located in the range of latitudes $\pm 30^{\circ}$, while outside that range most loops showed an increase of temperature with height, the so-called "up" loops. Independent observational evidence for down loops in the same range of heights of our analysis $\left(1.0-1.2 R_{\odot}\right)$ has been recently found by Prasad et al. (2013), who analyzed several temperature-sensitive line ratios in off-limb UV spectra and found decreasing temperatures with height.

From a theoretical perspective, hydrostatic (HS) down loops can be expected if the heating is strongly localized in the footpoint and the apex is mostly heated by electron heat conduction (Aschwanden \& Schrijver 2002; Serio et al. 1981). If the heating is assumed to have an exponential dependence on height, down loops can be expected if the heating scale height $s_{H}$ is much smaller than the loop length $L$ (Aschwanden \& Schrijver 2002). The latitudinal distribution of down loops and up loops found in Paper I indicates that the heating scale length $s_{H}$ varies with latitude, being smaller in the low-latitude regions. Similar mechanisms as those causing wave damping at low heights in coronal holes (Hahn et al. 2012) could be operating in the low-latitude region within the streamer belt, where the down loop population is observed. The down loop population seen in CR-2077 may be related to the relatively simpler configuration of the magnetic field (mainly dipolar and quadrupolar), as well as to the possibly weaker magnetic strengths, that characterized that specific rotation, and how Alfvén waves are dissipated in inhomogeneous fields. A natural question follows: is the down loop population strongly dependent on the level of activity and complexity of the solar corona? This query motivated the present study in which we apply the MLDT analysis to 11 rotations that sample the last minimum and the surrounding descending and ascending phases belonging to SC-23 and SC-24, respectively. As we will show, down loops are characteristic of the deep minimum, being much less observed from this time, supporting the idea of heating mechanisms being modulated by the evolving coronal magnetic field.

\section{MLDT ANALYSIS AND EUV DATA}

To analyze the evolution of the up/down distributions around the solar minimum, in this work we extended the study of Paper I to 11 rotations between CR-2055 and CR-2106. New to the analysis for each rotation, we compared the up/down populations to sunspot numbers. Figure 1 shows the monthly sunspot number (black dots) for SC-23 and SC-24 provided by the National Oceanic and Atmospheric Administration (NOAA) Space Weather Prediction Center. The blue solid curve is the 


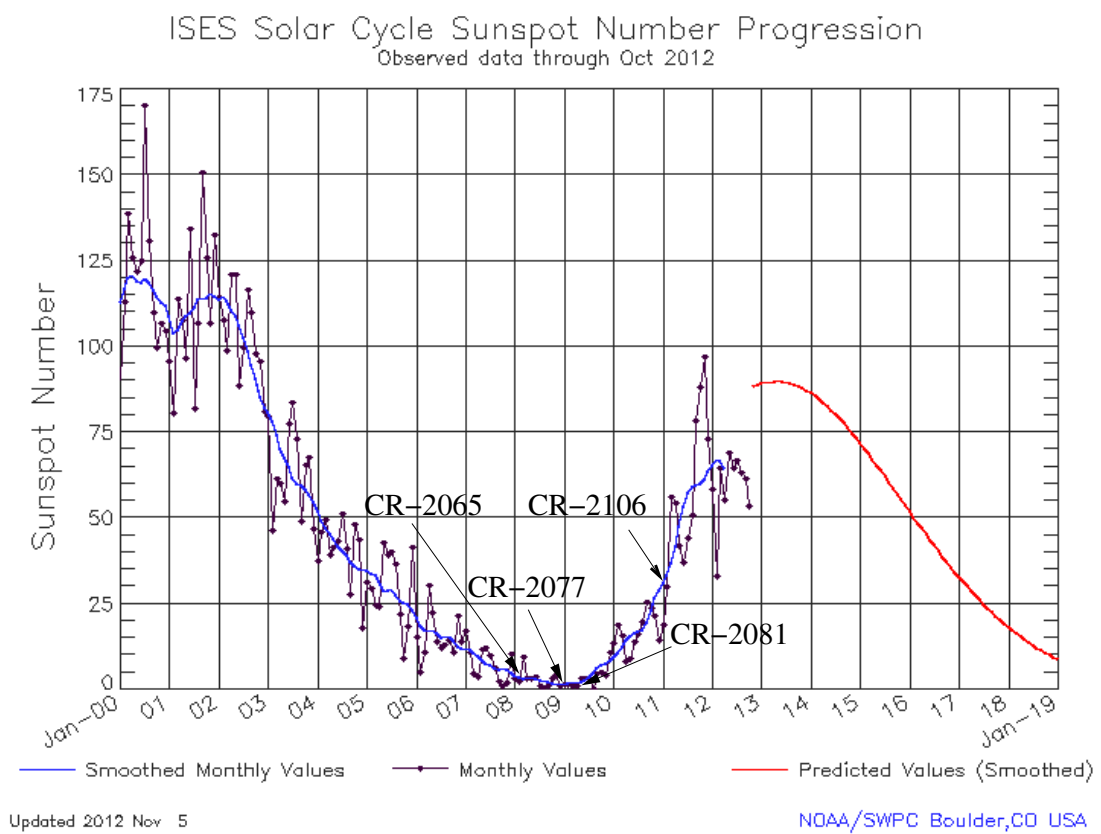

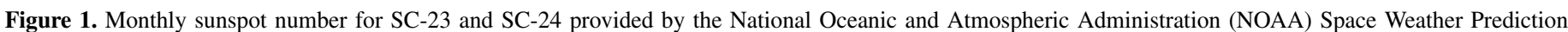

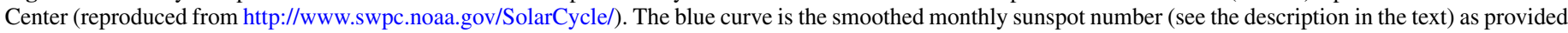
by the International Space Environment Service (ISES). We indicate the data points that correspond to four rotations for which we performed the MLDT analysis.

(A color version of this figure is available in the online journal.)

smoothed monthly sunspot number as provided by the International Space Environment Service (ISES). The smoothed monthly sunspot value is obtained averaging all monthly values from six months before to six months after the current month (month 0 ), and then using a total of 13 months of data. All the weights have value unity, except for the outer months (months -6 and +6 ) that have a value of $1 / 2$. This algorithm provides the monthly number using the yearly scale trend, which is useful to evaluate the monthly data in the context of a longer timescale tendency.

With these sunspot counts as a contextual reference, we analyzed the following 11 CRs: 2055, 2065, 2068, 2071, 2074, 2077, 2078, 2081, 2084, 2099, and 2106. The first one corresponds to 2007 April, while the last one corresponds to 2011 January and February. There was a 1 yr long period that showed a near-zero count (less than three) in the smoothed monthly sunspot number, from 2008 July to 2009 June (see Figure 1). Hereafter, we will refer to this period as the "deep minimum." Five of the rotations that we analyzed were chosen to be within and around that period; they are: CR-2071 (2008 June and July), CR-2077 and CR-2078 (2008 November-2009 January), CR-2081 (2009 March and April), and CR-2084 (2009 June). Rotations 2077, 2078, and 2081 sample the center of the deep-minimum period. Rotations 2071 and 2084 sample the end of the descending phase and the beginning of the subsequent ascending phase, respectively.

\subsection{MLDT}

The MLDT combines tomographic reconstructions (DEMT) of the three-dimensional distribution of the DEM with a magnetic model of the global corona. So far, only potential field source surface (PFSS) models have been used. The combination of the DEMT products with the PFSS models allows the determination of the electron density and electron temperature along individual magnetic field lines for the global corona. This is achieved by tracing individual field lines of the PFSS model and evaluating the DEMT products along them. The DEMT technique is explained in detail in Frazin et al. (2009); here we briefly summarize its central points. The technique consists of two consecutive procedures. In the first step, a time series of EUV images spanning a full solar synodic rotation is used to perform a solar rotational tomography (SRT) for each band of the EUV telescope separately. ${ }^{3}$ The product of the tomographic inversion in each band is the three-dimensional distribution of the filter band emissivity (FBE), defined as the wavelength integral of the coronal EUV spectral emissivity and the telescope's passband function of each band.

In the second step, the FBE values obtained for all bands in each tomographic cell (or voxel) are used to constrain the determination of a local differential emission measure (LDEM) distribution. The LDEM distribution $\xi(T)$, a function of temperature, is similar to the standard DEM, but it describes exclusively the plasma contained in the respective tomographic voxel, greatly mitigating the line of sight ambiguity that is intrinsic to standard DEM analysis. In exchange, the LDEM lacks the temporal resolution of the standard DEM, as it is based on time series of images spanning a full solar rotation. The LDEM analysis is then reliable for coronal regions that exhibit stability during their transit on the solar disk. For such regions, the LDEM is a measure of the thermal distribution of the plasma. Its zeroth and first moments are the mean squared electron density $\left(\left\langle N_{e}^{2}\right\rangle\right)$ and electron mean temperature $\left(T_{m}\right)$, respectively, where the average is taken over the LDEM distribution:

$$
\left\langle N_{e}^{2}\right\rangle \equiv \int d T \xi(T)
$$

\footnotetext{
3 SRT is put forth as a global optimization problem. The objective function is a sum of the quadratic norm of the difference between real and synthesized images, and a regularization (smoothing) term controlled by a single regularization parameter $p$. The regularization level is determined through cross-validation of the resulting model with complementary images not used for the SRT (Frazin et al. 2009; Vásquez et al. 2010).
} 


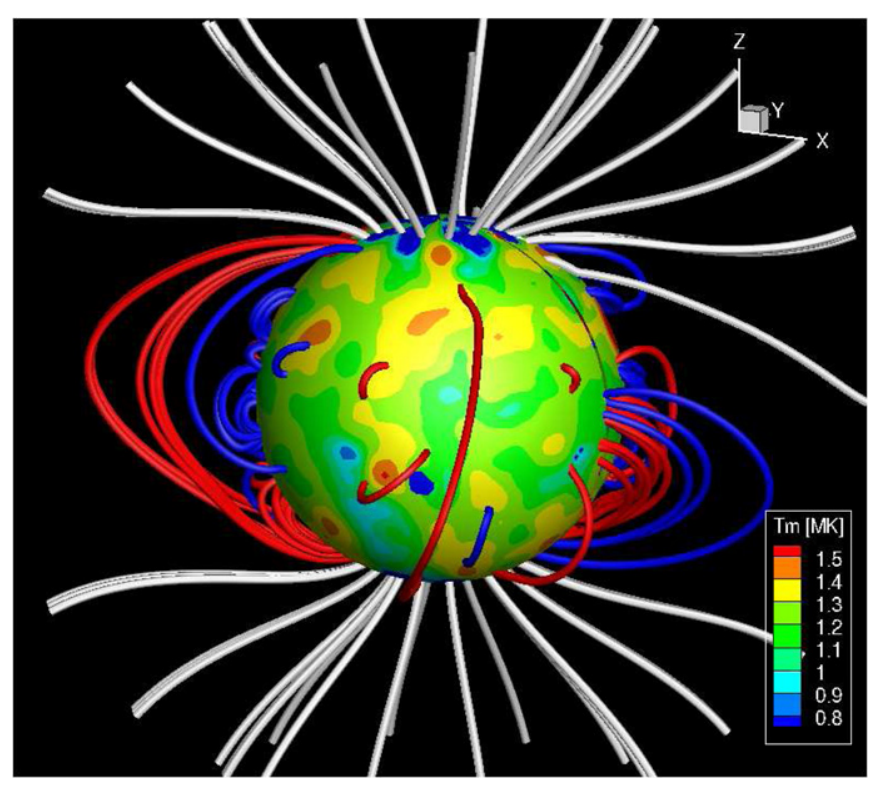

Figure 2. Three-dimensional representation of the up and down loop geometry for CR-2081, with red and blue depicting up and down loops, respectively. The central Carrington longitude was $118^{\circ}$. The spherical surface has a radius at $1.035 R_{\odot}$ and shows the LDEM electron temperature $T_{m}$ according to the color scale.

(A color version of this figure is available in the online journal.)

$$
T_{m} \equiv \frac{1}{\left\langle N_{e}^{2}\right\rangle} \int d T \xi(T) T .
$$

The tomographic grid covers the height range $1.00-1.25 R_{\odot}$ with a radial resolution of $0.01 R_{\odot}$ and an angular resolution of $2^{\circ} \times 2^{\circ}$. Due to optical depth issues at the base of the tomographic grid, EUV signal-to-noise levels, and scattered light effects (Shearer et al. 2012), the DEMT results are generally reliable in the height range from 1.03 to $1.20 R_{\odot}$. For the present work, we use data from this height range only. At these heights, the coronal magnetic field is globally well described by PFSS models (Riley et al. 2006), except where coronal currents may be high, as is possible near filaments, for example. In this work, we use the PFSS model developed by Tóth et al. (2011), which uses a finite-difference solver to calculate the magnetic field, thus providing a more accurate result for high-latitude regions than are typically obtained with spherical harmonic expansion methods.

Each closed magnetic loop of the PFSS model is divided into two legs, defined as the two segments that connect the loop apex with the photosphere. The DEMT temperature $T_{m}(r)$ is traced along each leg through the tomographic grid, where $r$ is the heliocentric height, and the data are fitted with a linear function $T_{m}(r)=a r+b$. The slope of the linear fit $a$ and the intercept $b$ are the two free fitting parameters evaluated for each leg. The parameter $a$ is then the fitted temperature gradient $d T_{m} / d r$ along each leg of a loop in the height range covered by the tomography. It is the sign of $a$ of each leg of the loop that is used to classify it into the up or down category. Also, only loops having the same sign of $a$ in both legs are counted. For each leg, the DEMT electron density is also traced and an HS fit is applied to the data. Thus, the base electron density $N_{0}$ and the density scale height $\lambda_{N}$ are also determined for each leg of all loops. These procedures are explained in Paper I.

To trace the field lines from the PFSS model through the tomographic solution of each rotation, we generated one starting

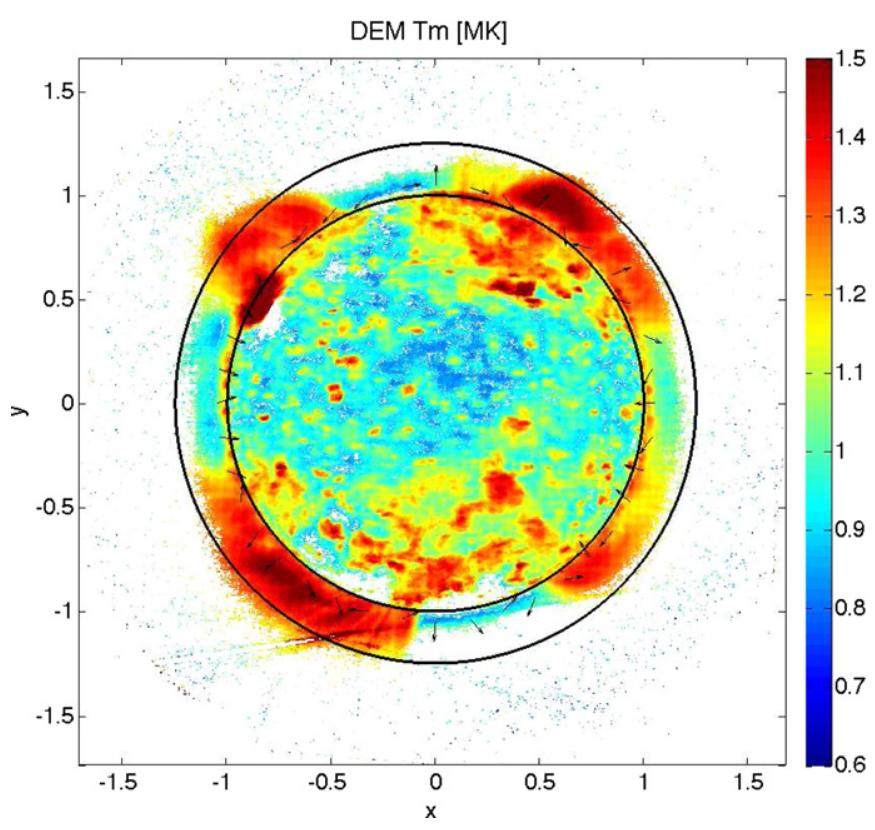

Figure 3. Determination of the DEM without tomography from six hourly images taken by EUVI-B between 6:00 and 12:00 UT on 2009 March 24 (CR-2081) corresponding to the same angle of vision as in Figure 2. Displayed is the mean of the DEM $T_{m}$, which corresponds to the average electron temperature along the line of sight. The black arrows indicate the direction of the twodimensional gradient of $T_{m}$ in the image plane.

(A color version of this figure is available in the online journal.)

point at the center of each tomographic cell at a height $1.075 R_{\odot}$, i.e., every $2^{\circ}$ in latitude and longitude, for a total of 16,200 points. The magnetic field line of the PFSS model passing through each of these points was traced both outward and inward until the coronal base $\left(1.0 R_{\odot}\right)$ and/or the source surface $\left(2.5 R_{\odot}\right)$. Only one point per tomographic cell was then kept. This point of the magnetic field line was assigned the values of the DEMT products $T_{m}$ and $N_{e}$ corresponding to the tomographic cell where it is located. The DEMT products along each closed field line were then separately grouped into the two legs. The linear fit was then applied to the $T_{m}(r)$ values of each leg separately, and the quality of each fit was measured by computing its coefficient of determination $R^{2}{ }^{4}$ As in Paper I, we based our analysis only on those field lines for which all the following conditions hold.

1. The field line is closed according to the PFSS model.

2. The temperature gradient of the linear fit to the temperature of both legs of the loop has the same sign. ${ }^{5}$

3. The quality of the linear fit is $R^{2}>0.5$ for both legs of the loop.

4. Both legs of the loop go through at least five tomographic grid cells with usable data.

As an example, a three-dimensional view of both up (red) and down (blue) loops for CR-2081 is displayed in Figure 2. For the same rotation, Figure 3 is an image of the mean temperature $T_{m}$ for CR-2081, corresponding to the same view angle as Figure 2. In the case of this $2 \mathrm{D}$ image, a DEM inversion is

\footnotetext{
$R^{2} \equiv 1-S_{\text {res }} / S_{\text {tot }}$, where $S_{\text {res }}$ is the sum of the squared residuals and $S_{\text {tot }}$ is the sum of data deviations from the mean.

5 For the different rotations we analyze in this paper, the fraction of closed loops that are excluded from the analysis by this second requirement ranges between $8 \%$ and $17 \%$. The different signs maybe due to real physical differences or unresolved dynamics.
} 
performed at each pixel, using the same technique applied for the inversion of the LDEM (Vásquez et al. 2010), but determined from the simultaneous images taken in the three coronal bands of EUVI-B. The mean temperature $T_{m}$ of each pixel is then computed as in Equation (2), but using the DEM instead of the LDEM. Overplotted in the image, the arrows indicate the two-dimensional temperature gradient at different locations. Downward gradients can be seen near the sector on both the $\mathrm{E}$ and $\mathrm{W}$ limbs, consistent with the up and down loop distributions. As in Paper I, this image shows that the presence of up and down loops is not an artifact of tomography.

As an example, Figure 4 shows the DEMT temperature profile along four loops and the corresponding least-squares fits to the data along the two legs of each loop. The examples include up and down loops, and for each case one example has an apex above $1.2 R_{\odot}$ (hereafter "large" loop) and another one below $1.2 R_{\odot}$ (hereafter "small" loop). We highlight this difference as we used the DEMT data up to $1.2 R_{\odot}$. Hence, small loops are fully contained in the DEMT grid, while large loops extend beyond the spatial range of the DEMT data. ${ }^{6}$ For the same loops shown in Figure 4, Figure 5 shows the corresponding HS fits to the DEMT electron density.

The pressure is also computed along each field line as $P=C N_{e} T_{m}$, where $C=k_{B}[(2+3 A) /(1+2 A)], A=$ $N(\mathrm{He}) / N(\mathrm{H}) \approx 0.08$ is the assumed ratio of helium and hydrogen abundances, and $N_{e}$ and $T_{m}$ are the local values from DEMT. We then apply an HS fit to the pressure data so that the base pressure $P_{0}$ and the scale height $\lambda_{P}$ are determined for each leg of all loops. Using the pressure $P$ and the PFSS magnetic field, we computed the plasma parameter $\beta=P / P_{\mathrm{mag}}$, where $P_{\text {mag }}=B^{2} / 8 \pi$ along each field line.

\subsection{Data Sources}

For each rotation, the DEMT analysis was performed with images taken by the Extreme UltraViolet Imager (EUVI) instrument aboard the B spacecraft of the Solar Terrestrial Relations Observatory (STEREO), and the PFSS model was computed using the corresponding synoptic magnetogram from the Solar and Heliospheric Observatory (SoHO) Michelson Doppler Imager (MDI) as a boundary condition. Since the final MDI synoptic data correspond to CR-2104, we used the synoptic magnetogram provided by the National Solar Observatory Global Oscillation Network Group (GONG) for CR-2106. We compared the MLDT results using alternative PFSS models based on either MDI or GONG magnetograms for rotations for which both sources were available, specifically for CR-2065 of the declining phase of SC-23 and for CR-2078 of the deep minimum. In tracing the DEMT results along the field lines, we found no significant differences in the statistics of the results, clearly indicating very similar geometries for the magnetic field in both models. There is a systematic shift of the GONG magnetic field strength to lower values when compared to the MDI magnetograms. This implies a shift to larger values of the plasma $\beta$ for the GONG based MLDT results, not changing the relative results for the up and down populations. Once corrected for the shift in magnetic field strength, the GONG based MLDT results are in agreement with those based on MDI magnetograms. We defer to a future effort details on the corrections required when changing between the different magnetogram sources, also including results based on the Helioseismic and Magnetic Imager

\footnotetext{
6 More than $70 \%$ of the loops that are rejected from the analysis due to having
} different signs of the temperature gradient in both legs are of the large kind. on board the Solar Dynamics Observatory. For the purposes of this work we do not apply such corrections, and it suffices to say that our results are statistically robust to the choice of magnetogram source.

\subsection{Regularization Level of the Tomography}

For all tomographic reconstructions, we set the tomographic regularization parameter at $p=1.0$, a typical value for the size of the images and the tomographic grid we used (Frazin et al. 2009). Before detailing our main results in the next section, we anticipate that in the case of CR-2077 they are very similar to those published previously in Paper I. The cause for slight differences is twofold: (1) a different value for the regularization parameter of the tomographic reconstruction was used, and (2) a different polar correction for the magnetic field of the PFSS model was used. The differences are not statistically significant and thus the MLDT results are robust to both effects.

In this work, we took the chance to further investigate the effect of the regularization level on the MLDT results. For CR-2065, CR-2081, CR-2084, and CR-2106, which sample the deep minimum and the ascending and descending phases, we computed the uncertainty in the fraction of up and down loops due to the regularization level of the tomographic reconstructions. For each of these rotations we varied the regularization parameter value in a range of appropriate values $p=1.0 \pm 0.5$ (Frazin et al. 2009; Vásquez et al. 2010, 2011). For each rotation, we computed the fraction of down and up loops for the different regularization levels, and used these values to compute their uncertainty, which we found to be of order $8 \%$. In addition, Paper I showed that the statistical results of the MLDT technique are robust to the uncertainty of the radiometric calibration of the EUVI channels.

\section{RESULTS}

In this section, we show detailed results for two rotations of the deep-minimum period, specifically CR-2077 and CR2081, and also for two rotations that sample the descending and ascending phases, namely, CR-2065 and CR-2106, respectively. We also present a summary of the main results for all the 11 rotations we analyzed.

Figure 6 shows results of the DEMT and MLDT studies concerning the temperature structure of the global corona for two rotations of the deep minimum, namely, CR-2077 (left panels) and CR-2081 (right panels). The top panels show Carrington maps of the DEMT $T_{m}$ at a tomographic grid height $1.075 R_{\odot}$. We overplot PFSSM magnetic strength $B$ contour levels (solid thin black and white curves) as well as the magnetically open/closed region boundaries (solid thick black curves). The black regions correspond to locations undetermined by the tomographic reconstruction due to dynamics (called zero density artifacts; see Frazin et al. 2009), and the white regions represent voxels for which the LDEM model does not successfully reproduce the tomographically reconstructed emissivities (see Nuevo et al. 2012; Vásquez et al. 2012). The middle panels show the spatial distribution of the up (orange and dark red) and down (light and dark blue) loops at $1.075 R_{\odot}$, and the bottom panels show the same at $1.0 R_{\odot}$. In the middle and bottom panels, dark blue and dark red represent regions threaded by large loops, while light blue and orange represent small loops. In the top and middle panels, the thick black curves indicate the open/closed boundary at $1.075 R_{\odot}$, and in the bottom panels the thick curves indicate the open/closed boundary at $1.0 R_{\odot}$. 

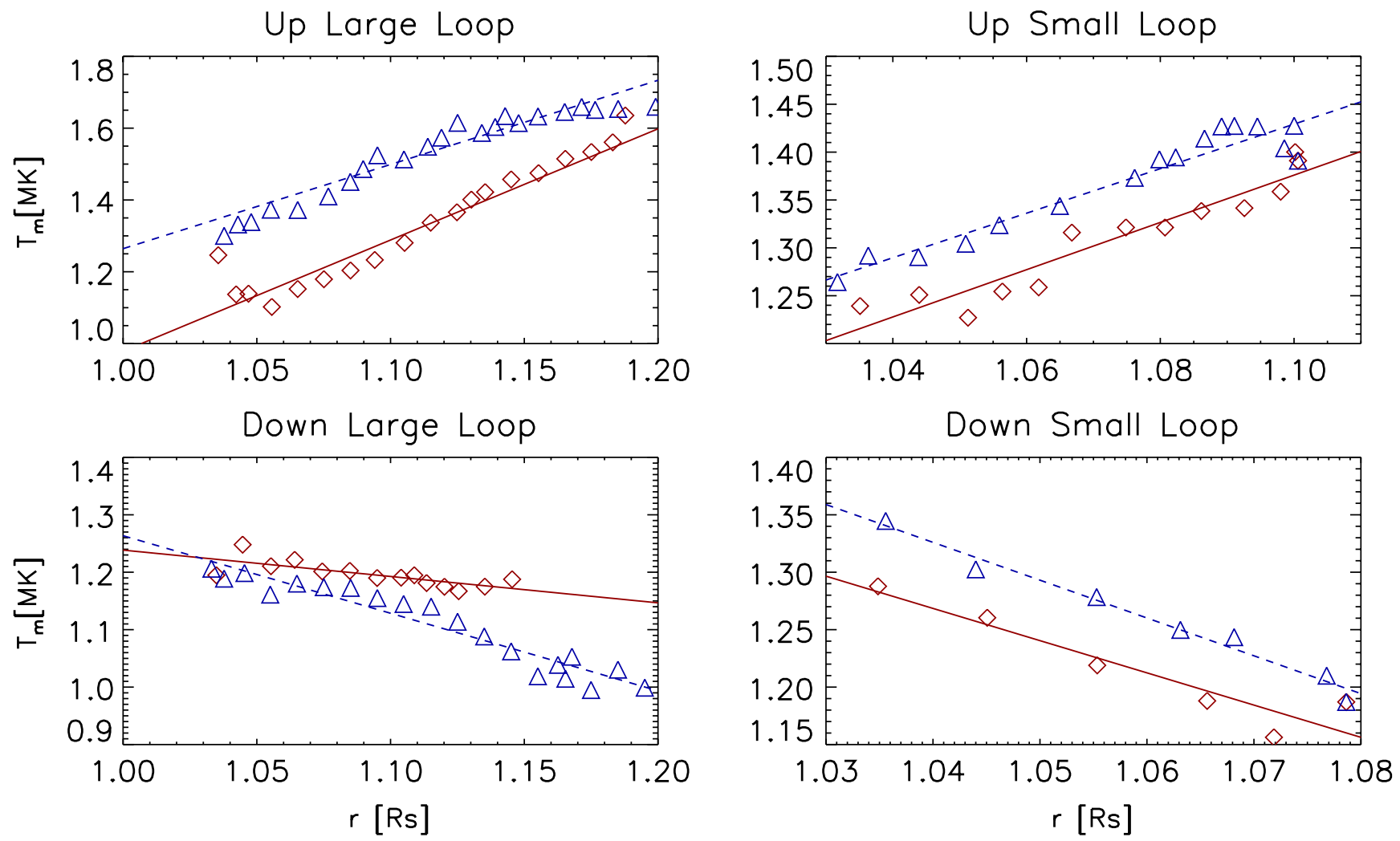

Figure 4. Least-squares fits of the form $T_{m}=a r+b$ used to classify loops as "up" $(a>0)$ or "down" $(a<0)$. Each panel shows the fits to each of the two legs of a selected loop, with red diamonds representing the DEMT $T_{m}$ values of one leg and blue triangles representing the values along the other one. The top panels show two examples of up loops, with the left panel showing a large case (with apex above $1.2 R_{\odot}$ ) and the right one showing a small case (with apex below $1.2 R_{\odot}$ ). The bottom panels show two examples of down loops, a large one (left panel) and a small one (right panel).

(A color version of this figure is available in the online journal.)
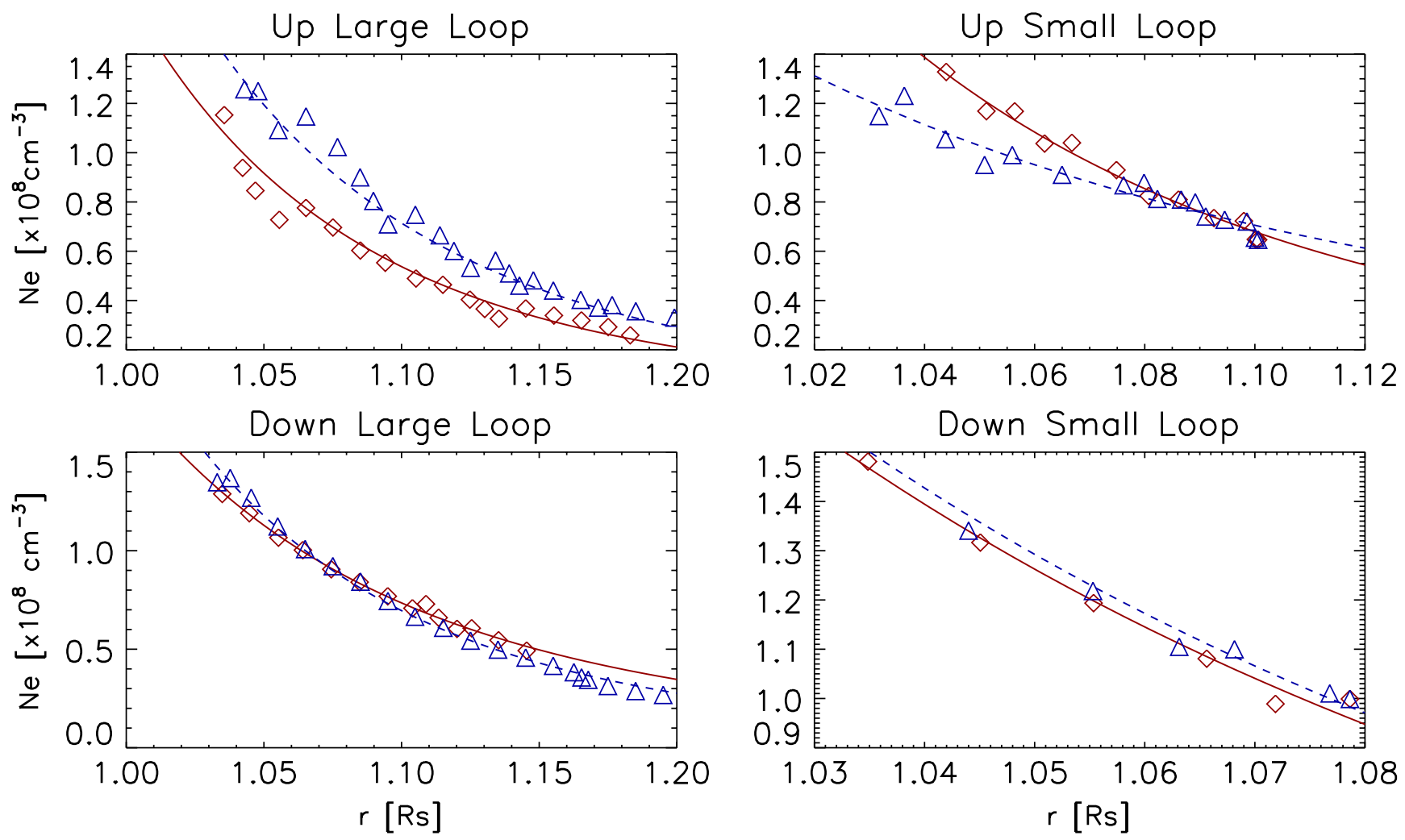

Figure 5. For the same four loops selected in Figure 4, these panels show the respective HS fits to the DEMT electron density along the two legs of each loop. The HS fits allow the determination of the base density $N_{0}$ and scale height $\lambda_{N}$ for each loop.

(A color version of this figure is available in the online journal.) 

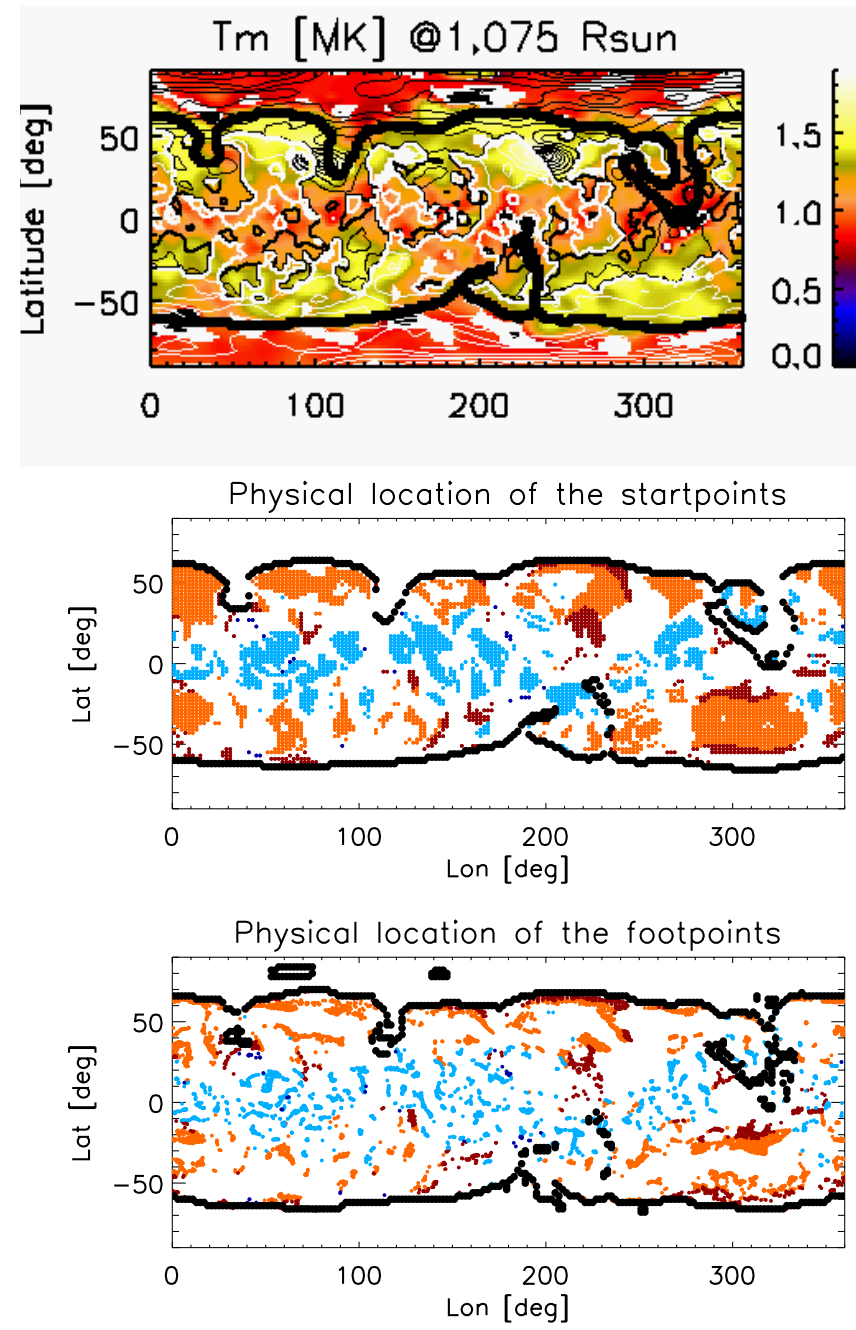
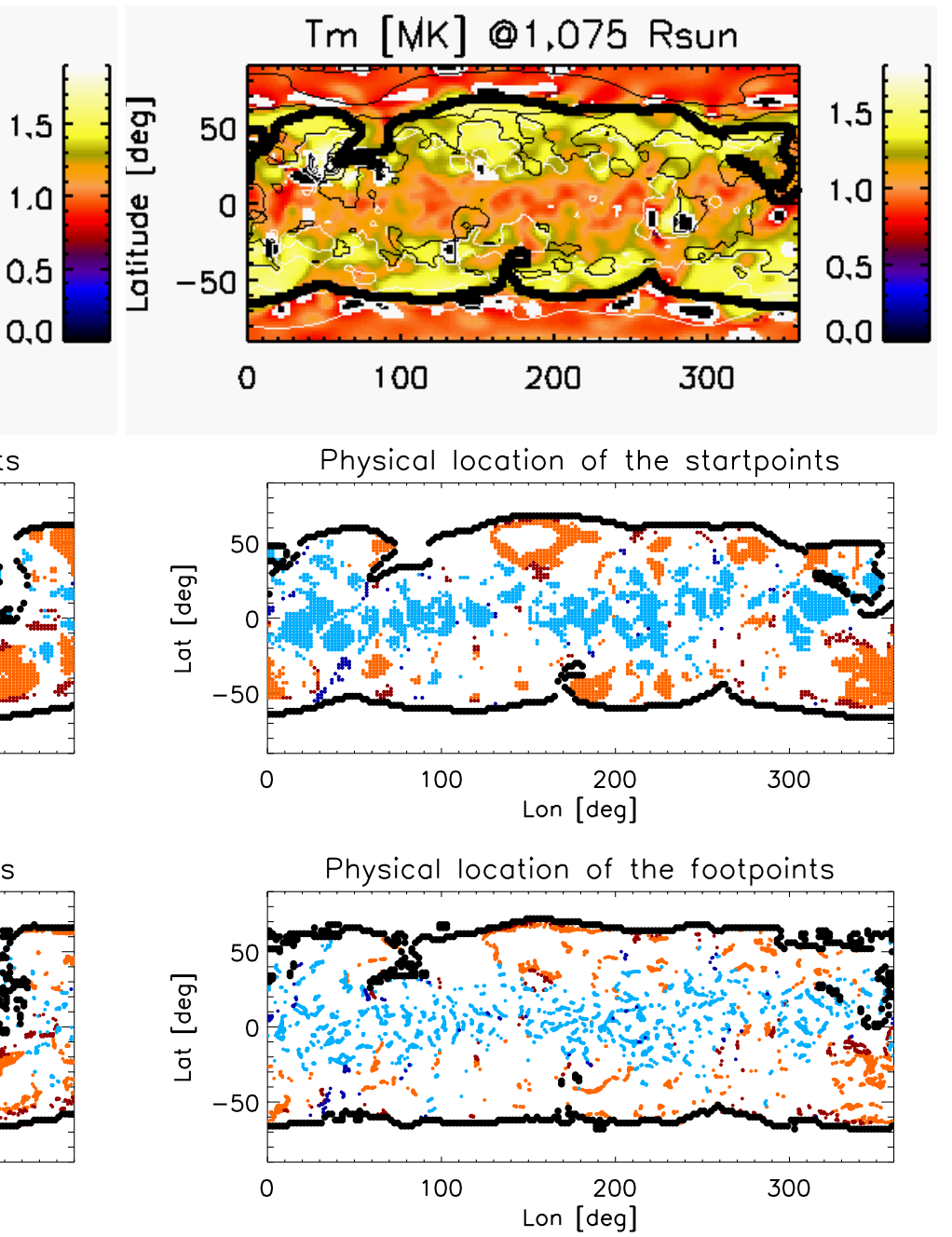

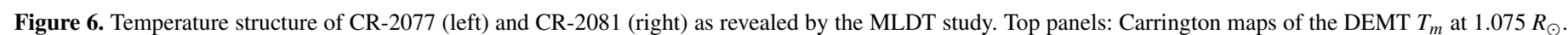

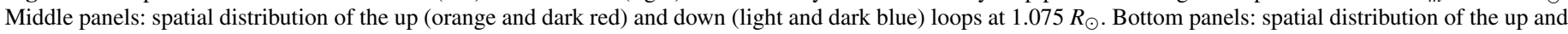

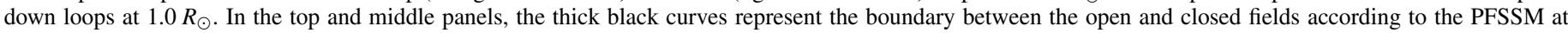

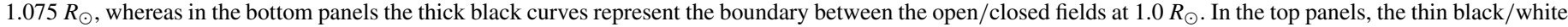

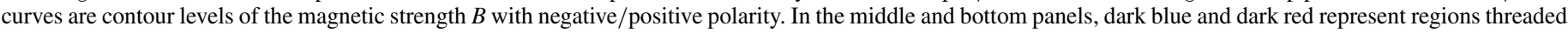

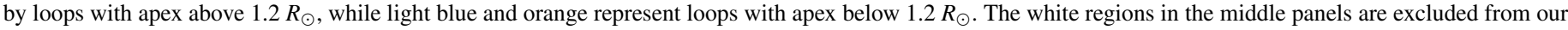
analysis as they are threaded by loops that do not meet all conditions listed in Section 2.

(A color version of this figure is available in the online journal.)

Figure 6 shows that, at the deep minimum, down loops are ubiquitous at low latitudes, while the up loops are located at intermediate latitudes, nearer to the open/closed boundary. The location and number of up and down loops are quantified in Figure 7. For CR-2077 (left) and 2081 (right), the top panels show frequency histograms of the fitted temperature gradient of the loops of Figure 6, measuring the relative fractions of the up and down loop populations. In the same figure, the middle and bottom panels show the frequency histograms of the latitudinal distribution of the up and down loops, respectively. The mean latitude of the down and up loops is located within $3^{\circ}$ of the equator in all cases. The distribution of down loops is unimodal and its small mean latitude is consistent with how they are distributed at low latitudes near the equator. The distribution of up loops is bi-modal, with one peak at a mid-latitude in each hemisphere, so that its small mean latitude summarizes how they are symmetrically distributed around the equator.

Figure 8 is similar to Figure 6 but for rotations CR-2065 (left panels) and CR-2106 (right panels), farther from the deep minimum, during the declining phase of SC-23 and the ascending phase of SC-24, respectively. It is readily seen that the down loop population is diminished when compared to the deep minimum rotations, while the up loop population is enhanced. The latitudinal distributions of up and down loops are similar to those during the deep minimum. This is shown in the histograms of Figure 9. The mean latitude is within $6^{\circ}$ of the equator for down loops and within $15^{\circ}$ for up loops. These larger departures from the equator, when compared to the deep minimum rotations, are consistent with the progressive loss of symmetry in the coronal structure during these more active rotations (specially for CR-2106 that shows the largest departure from the equator).

Comparing the three panels of Figures 6 and 8, we note that the up loops tend to be somewhat hotter than the down loops. Quantitative information for these trends is shown in Figure 10, displaying the scatter plot of the fitted temperature gradient $d T_{m} / d r$ versus the average temperature $T_{m}$ along each leg $\left(\left\langle T_{m}\right\rangle\right)$ for each rotation. For the deep minimum rotations CR-2077 and CR-2081, the mean ratio of the average temperature of the up loops to that of the down loops is 1.26 and 1.23 , respectively. 

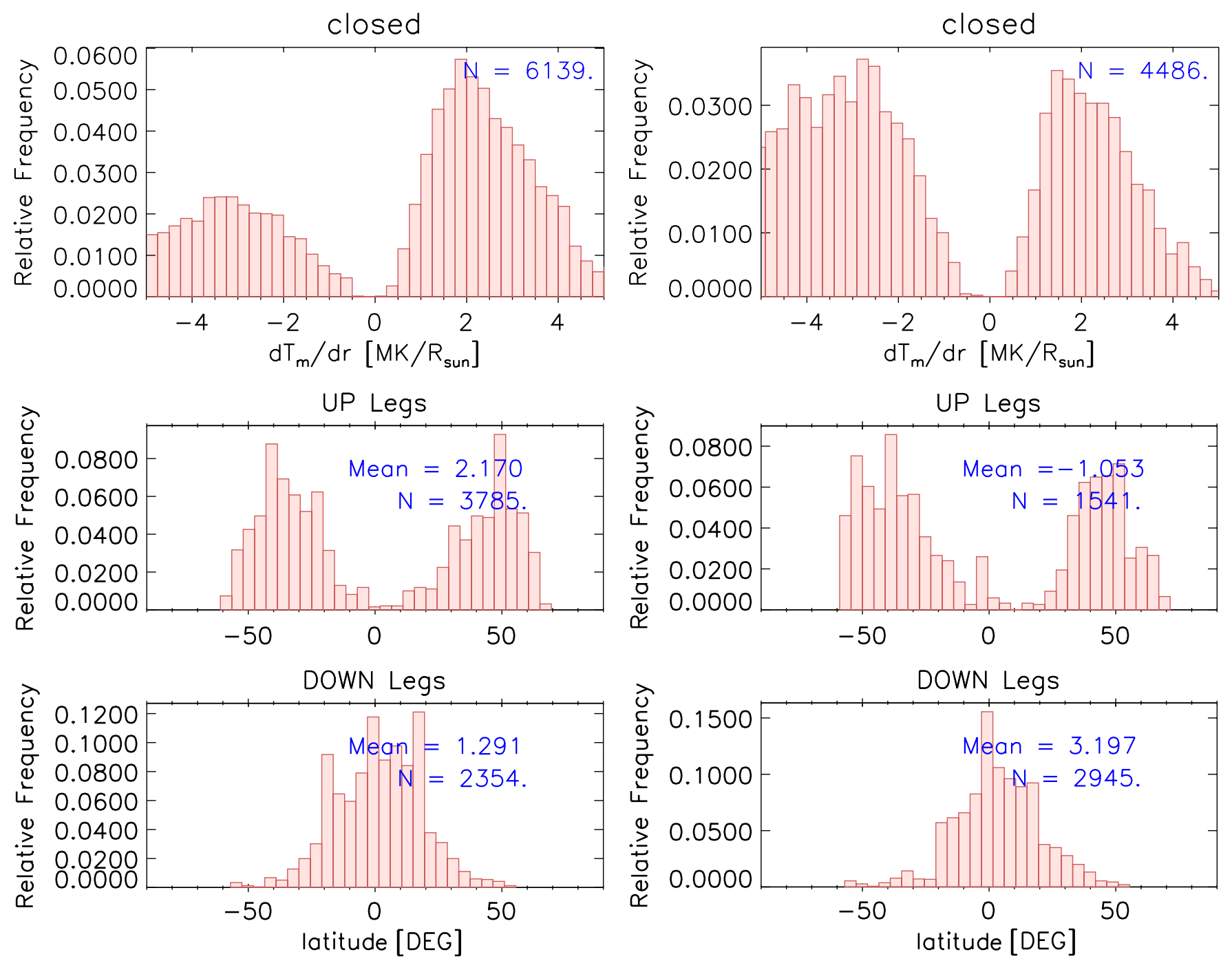

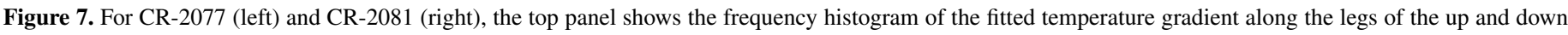

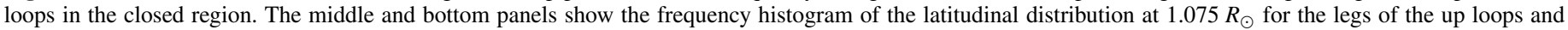
down loops, respectively.

(A color version of this figure is available in the online journal.)

Noting that the most active regions of the corona exhibit higher temperatures, a comparison of the top and middle panels of these figures also indicates that down loops are mostly located in the QS regions. The up loops are located in both active and QS regions.

Table 1 summarizes statistical information of the four rotations shown in Figures 6-9. All quantities are calculated separately for up and down loops, as well as for large and small ones. We tabulate the total number of legs, indicating the fraction of them that are localized in low latitudes (within the $\pm 30^{\circ}$ band) and middle latitudes (outside that band), as well as the total loop length. We also tabulate the average value for all legs of the parameters of the HS fits, namely, the base values and scale height of the density $\left(N_{0}, \lambda_{N}\right)$ and the pressure $\left(P_{0}, \lambda_{P}\right)$. Finally, we tabulate the average value of the fitted temperature gradient $a=d T_{m} / d r$.

We can now quantify the global distribution of both up and down loops. For all rotations, most up and down loops are small. The vast majority of the small down loops (more than $91 \%$ ) are located within the $\pm 30^{\circ}$ latitude range. Also, the percentage of small up loops located outside that latitude range is greater than
$75 \%$ in three rotations, and greater than $61 \%$ in one case. As for large loops, in general, more than half of their up population lies outside the $\pm 30^{\circ}$ latitude range, and the vast majority of their down population is within that range (except for CR-2106, for which the number of loops is very low).

We can highlight some differences between the different kinds of loops. As the pressure is a product of the density and the temperature, up loops are characterized by $\lambda_{P}>\lambda_{N}$, while the opposite happens for down loops, as explained in Paper I. While the average density scale height of up loops is somewhat larger than that of down ones for all rotations, the difference in the average pressure scale height of the up and down loops is noticeably larger (see Table 1). This is a consequence of $P \propto N T$ and the opposite behavior of $T(r)$ for up and down loops. The ratio of the mean pressure scale height of up loops to that of down loops for all rotations ranges between 1.6 and 1.9 for small and large loops, respectively. Figure 11 shows scatter plots of the fitted temperature gradient $d T_{m} / d r$ versus the pressure scale height $\lambda_{P}$ for the same four rotations shown in Figure 10. The up loops tend to be characterized by larger pressure scale heights than down loops, as expected due 

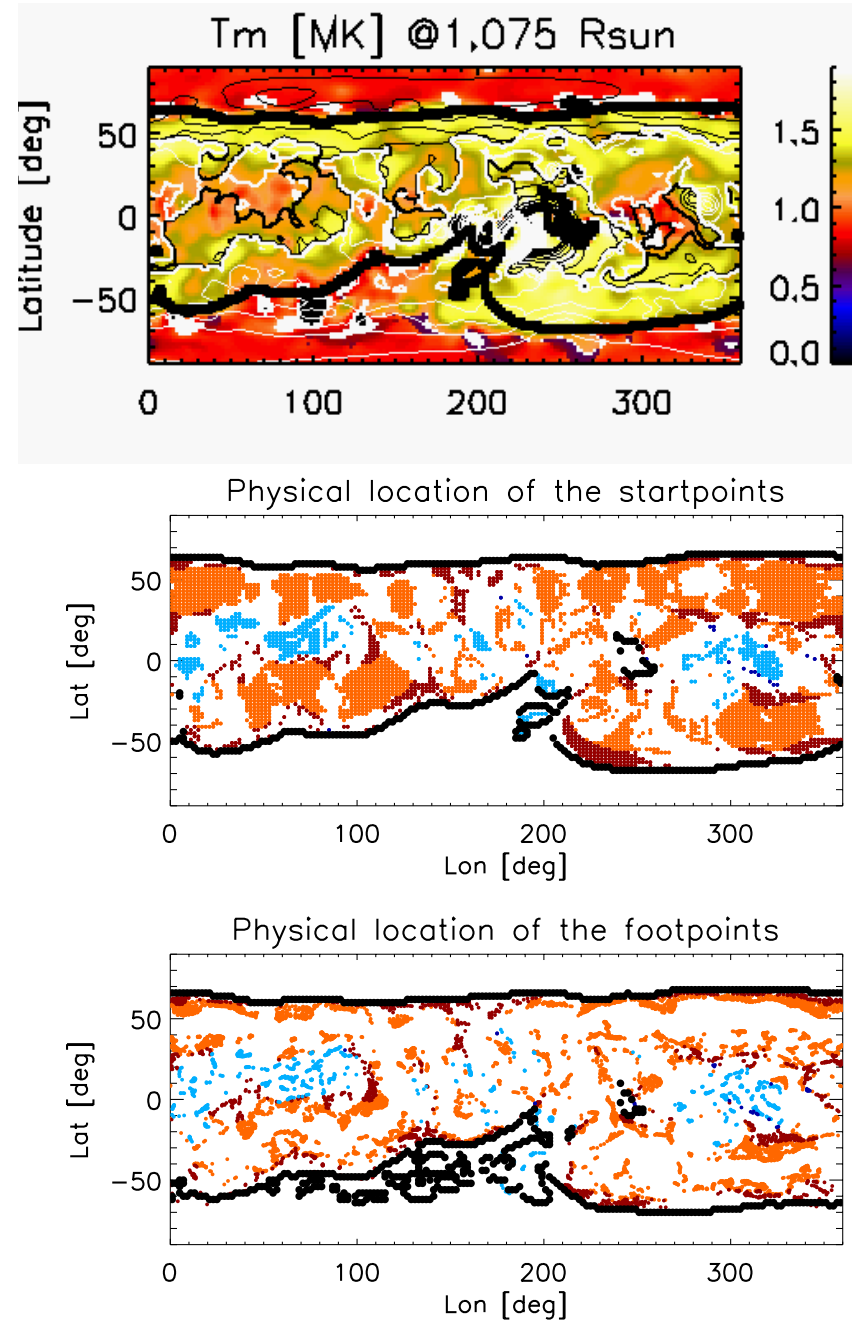

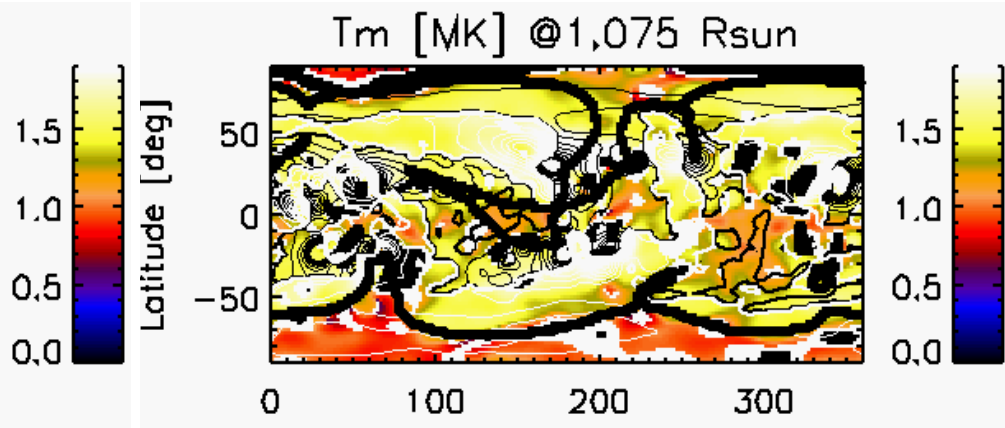

Physical location of the startpoints

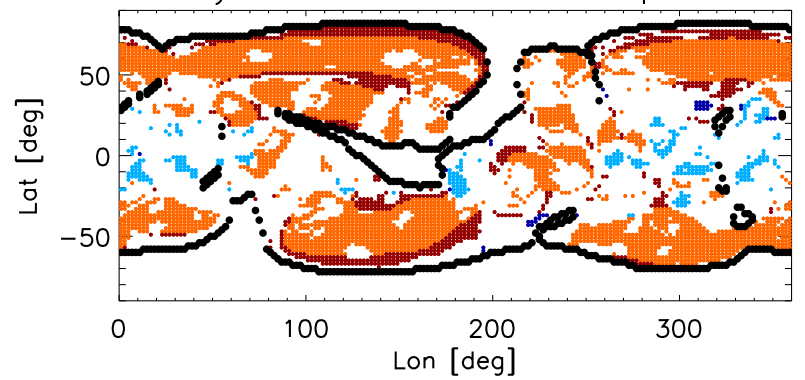

Physical location of the footpoints

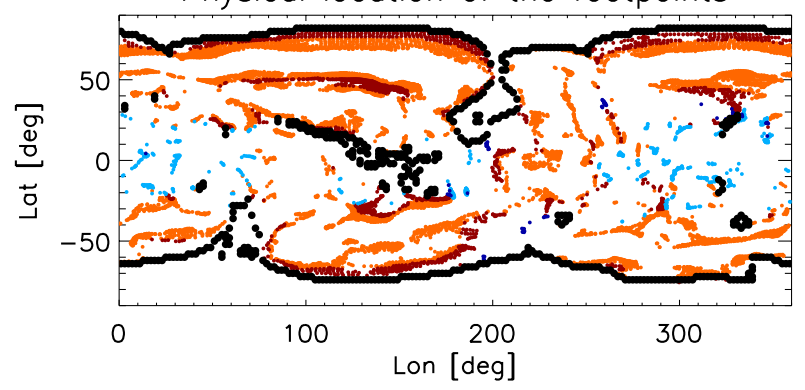

Figure 8. Same as Figure 6 but for rotation CR-2065 (left) and CR-2106 (right).

(A color version of this figure is available in the online journal.)

Table 1

Statistics of the Number of Magnetic Loop Legs Analyzed, their Latitudinal Distribution, and Loop Length, Discriminating the Different Types of Legs: Up, Down, Small, and Large

\begin{tabular}{|c|c|c|c|c|c|c|c|c|c|c|}
\hline $\mathrm{CR}$ & Leg Type & $\begin{array}{l}\text { Number } \\
\text { of Legs }\end{array}$ & $\begin{array}{l}\text { Percentage of } \\
\text { Footpoints within } \\
\pm 30^{\circ} \text { Latitude }\end{array}$ & $\begin{array}{l}\text { Percentage of } \\
\text { Footpoints Outside } \\
\pm 30^{\circ} \text { Latitude }\end{array}$ & $\begin{array}{l}\text { Average Loop } \\
\text { Length }\left(R_{\odot}\right)\end{array}$ & $\begin{array}{c}\text { Average } \\
N_{0}\left(10^{8} \mathrm{~cm}^{-3}\right)\end{array}$ & $\begin{array}{c}\text { Average } \\
P_{0}\left(10^{-3} \mathrm{~Pa}\right)\end{array}$ & $\begin{array}{l}\text { Average } \\
\lambda_{N}\left(R_{\odot}\right)\end{array}$ & $\begin{array}{l}\text { Average } \\
\lambda_{P}\left(R_{\odot}\right)\end{array}$ & $\begin{array}{c}\text { Average } \\
d T_{m} / d r \\
\left(\mathrm{MK} / R_{\odot}\right)\end{array}$ \\
\hline \multirow[t]{4}{*}{2065} & Small up & 4691 & 39 & 61 & 0.55 & 2.3 & 7.1 & 0.079 & 0.099 & 3.21 \\
\hline & Small down & 858 & 95 & 5 & 0.33 & 2.6 & 10.4 & 0.076 & 0.059 & -4.32 \\
\hline & Large up & 1276 & 53 & 47 & 1.51 & 2.2 & 7.2 & 0.095 & 0.114 & 1.98 \\
\hline & Large down & 32 & 91 & 9 & 1.23 & 2.4 & 9.5 & 0.076 & 0.063 & -3.26 \\
\hline \multirow[t]{4}{*}{2077} & Small up & 2990 & 25 & 75 & 0.52 & 2.2 & 7.0 & 0.083 & 0.103 & 2.81 \\
\hline & Small down & 49 & 76 & 24 & 1.55 & 2.3 & 8.5 & 0.077 & 0.068 & -2.04 \\
\hline & Large up & 795 & 44 & 56 & 1.52 & 1.9 & 5.9 & 0.097 & 0.119 & 1.99 \\
\hline & Large down & 2305 & 91 & 9 & 0.35 & 2.3 & 8.7 & 0.079 & 0.061 & -3.86 \\
\hline \multirow[t]{4}{*}{2081} & Small up & 1296 & 22 & 78 & 0.53 & 2.1 & 6.8 & 0.081 & 0.098 & 2.44 \\
\hline & Small down & 2802 & 93 & 7 & 0.37 & 2.4 & 9.5 & 0.078 & 0.059 & -4.39 \\
\hline & Large up & 245 & 42 & 58 & 1.6 & 1.9 & 6.0 & 0.093 & 0.108 & 1.64 \\
\hline & Large down & 143 & 60 & 40 & 1.79 & 2.4 & 10.2 & 0.083 & 0.069 & -2.93 \\
\hline \multirow[t]{4}{*}{2106} & Small up & 5017 & 12 & 88 & 0.5 & 2.3 & 8.2 & 0.084 & 0.108 & 3.65 \\
\hline & Small down & 426 & 99 & 1 & 0.44 & 2.5 & 11.1 & 0.073 & 0.058 & -4.68 \\
\hline & Large up & 1282 & 22 & 78 & 1.21 & 2.2 & 8.3 & 0.109 & 0.136 & 2.15 \\
\hline & Large down & 60 & 35 & 65 & 2.02 & 2.0 & 4.3 & 0.094 & 0.082 & -2.1 \\
\hline
\end{tabular}

Note. We also tabulate the average value of several quantities measured along the legs of the magnetic loops: the parameters $N_{0}$ and $\lambda_{N}$ are the base density and density scale height, respectively; $P_{0}$ and $\lambda_{P}$ are the base pressure and pressure scale height, respectively; and $d T_{m} / d r$ is the fitted temperature gradient. 

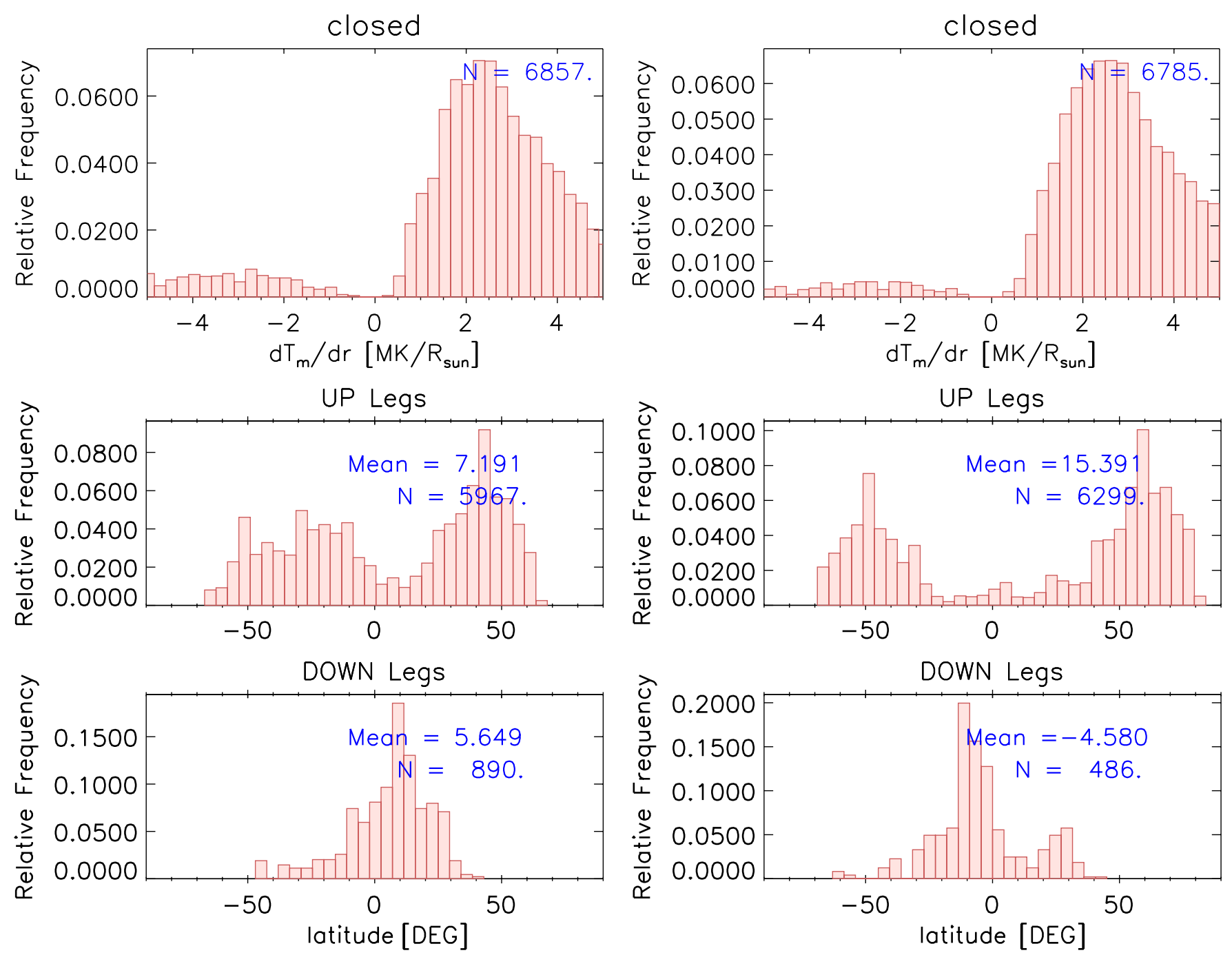

Figure 9. Same as Figure 7 but for rotation CR-2065 (left) and CR-2106 (right).

(A color version of this figure is available in the online journal.)

to the increase in temperature with height. For down loops, the relationship between the fitted temperature gradient and the pressure scale height is strongly linear, with correlation coefficients larger than 0.7 for all rotations. This is expected as a larger (but negative) temperature gradient yields a more quickly decreasing pressure and hence a smaller pressure scale height.

The average scale height of large loops is somewhat larger than that of small loops (see Table 1). For up loops, the ratio of the mean density scale height $\lambda_{N}$ of large loops to that of small ones is 1.20 , while the ratio of the respective mean pressure scale heights $\lambda_{P}$ is 1.17 . For down loops, the ratio of the mean density scale height $\lambda_{N}$ of large loops to that of small ones is 1.08, while the ratio of the respective mean pressure scale heights $\lambda_{P}$ is 1.18. More noticeable differences between large and small loops are to be found in the fitted temperature gradient $d T_{m} / d r$, with small loops showing stronger gradients (see Table 1). For up loops, the ratio of the mean fitted temperature gradient of small loops to that of large ones is 1.55 . For down loops, the ratio of the average fitted temperature gradient of small loops to that of large ones is 1.73. Finally, for the different rotations we analyzed around the solar minimum, we did not find a strong variability of the average value of the different fitting parameters.
Figure 12 shows scatter plots of the fitted temperature gradient $d T_{m} / d r$ versus the average value of the plasma parameter $\beta$ along each leg $(\langle\beta\rangle)$. There is a clear tendency for down loops to have $\beta \gtrsim 1$, while up loops tend to be characterized by $\beta<1$ values. Similar plots, made at the lowest reliable height of the tomographic grid, $1.035 R_{\odot}$, as well as at the apex of the loops, show the same trends. Scatter plots of $d T_{m} / d r$ versus the average magnetic field strength along each leg $\langle B\rangle$ and versus the average pressure along each leg $\langle P\rangle$ (not included in this paper) indicate that, while there are no significant differences in the average pressure between up and down loops, the magnetic strength is somewhat bigger for up loops. As $\beta \propto 1 / B^{2}$, the differences of $\beta$ are mainly due to differences in the magnetic strength, being smaller in down loops.

For the same rotations shown in Figure 10, Table 2 summarizes the median and standard deviation of the distribution of the mean plasma parameter $\langle\beta\rangle$ averaged between heights 1.035 and $1.20 R_{\odot}$ along each leg. The table also displays the statistics of the distribution of the $\beta$ value of each leg at $1.035 R_{\odot}$. The table discriminates the different types of loops: up, down, small, and large. We focus now on the small loops, which constitute the vast majority of the statistics. For all rotations, the small down loops are characterized by median $\langle\beta\rangle \gtrsim 1$, while up loops show 

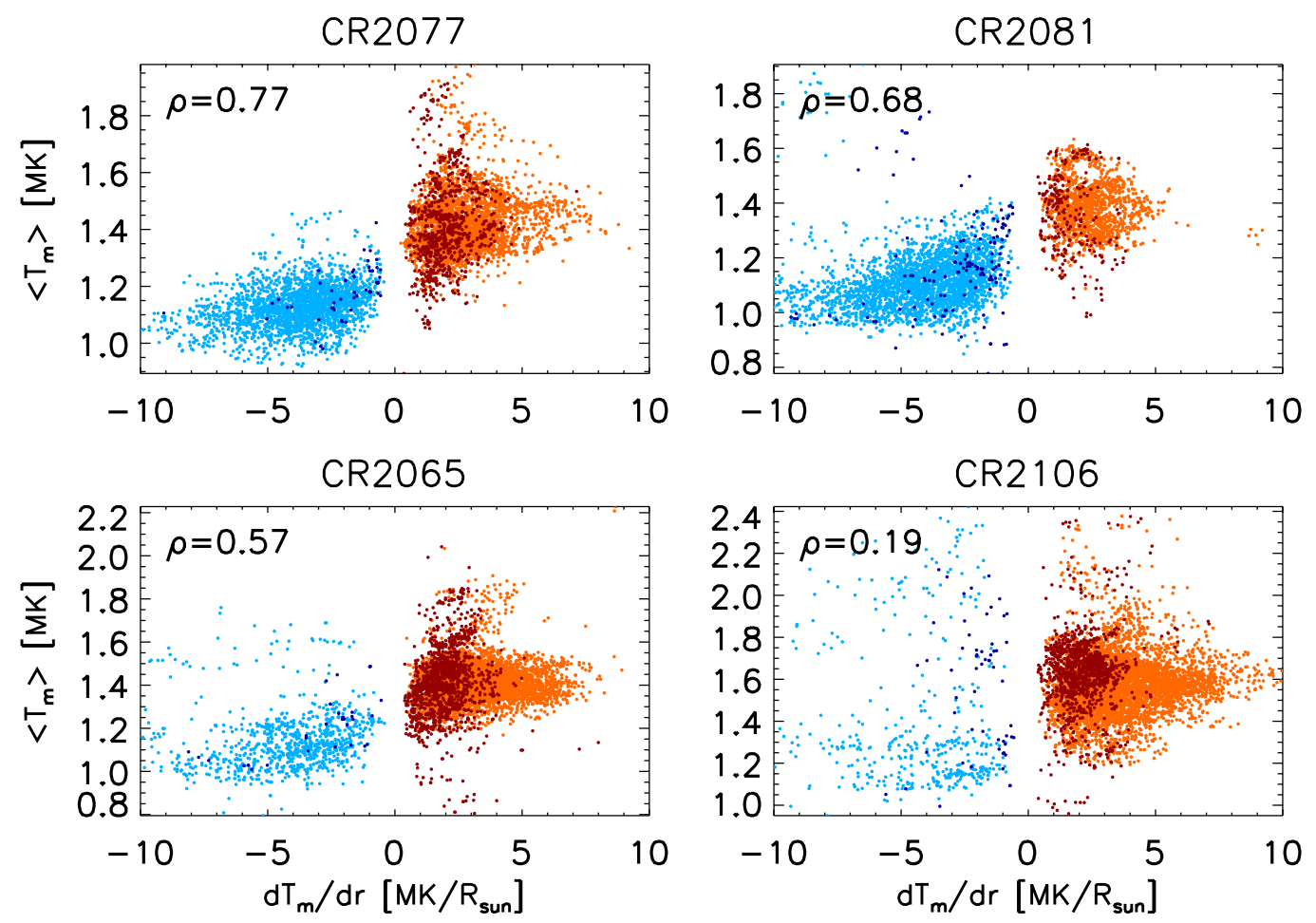

Figure 10. Scatter plots of the fitted temperature gradient $d T_{m} / d r$ vs. the average temperature along each leg $\left\langle T_{m}\right\rangle$ for the rotations shown in Figure 6 (top panels) and Figure 8 (bottom panels). In each scatter plot, the corresponding linear Pearson correlation coefficient of the whole population is indicated. The color code differentiates the four kinds of loops: up small (orange), up large (dark red), down small (light blue), and down large (dark blue).

(A color version of this figure is available in the online journal.)

CR2077
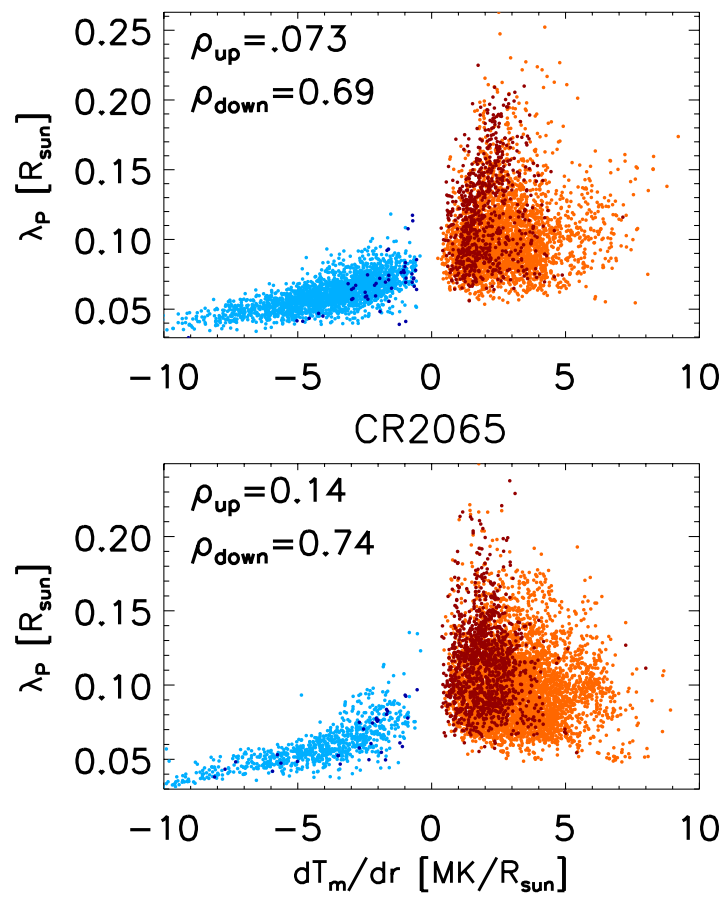

CR2081
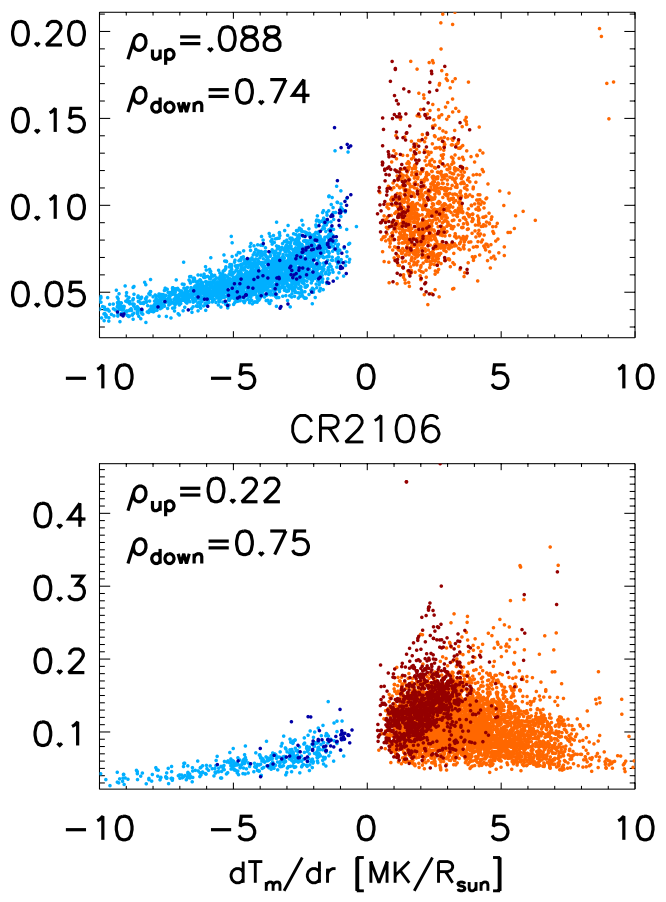

Figure 11. Scatter plots of the fitted temperature gradient $d T_{m} / d r$ vs. the pressure scale height $\lambda_{P}$, for the same rotations shown in Figure 10, using the same color code to distinguish the different populations of loops. The Pearson correlation coefficient of the up and down population is indicated for each rotation.

(A color version of this figure is available in the online journal.)

median $\langle\beta\rangle<1$. The scatter plots in Figure 12 and the standard deviations show that the small down and up populations are well differentiated, with virtually all down loops characterized by $\langle\beta\rangle>1$ and a majority of up loops by $\langle\beta\rangle<1$. The ratio of the median $\langle\beta\rangle$ of small down loops to that of small up loops ranges between 3 and 5 . The $\beta$ at the lowest height available in our analysis $\left(1.035 R_{\odot}\right)$ shows again the trend of larger values for the small down loops, their median $\beta$ being about twice that of the small up loops. Turning now to large loops, differences are somewhat smaller, but the same trends hold. The ratio of the 


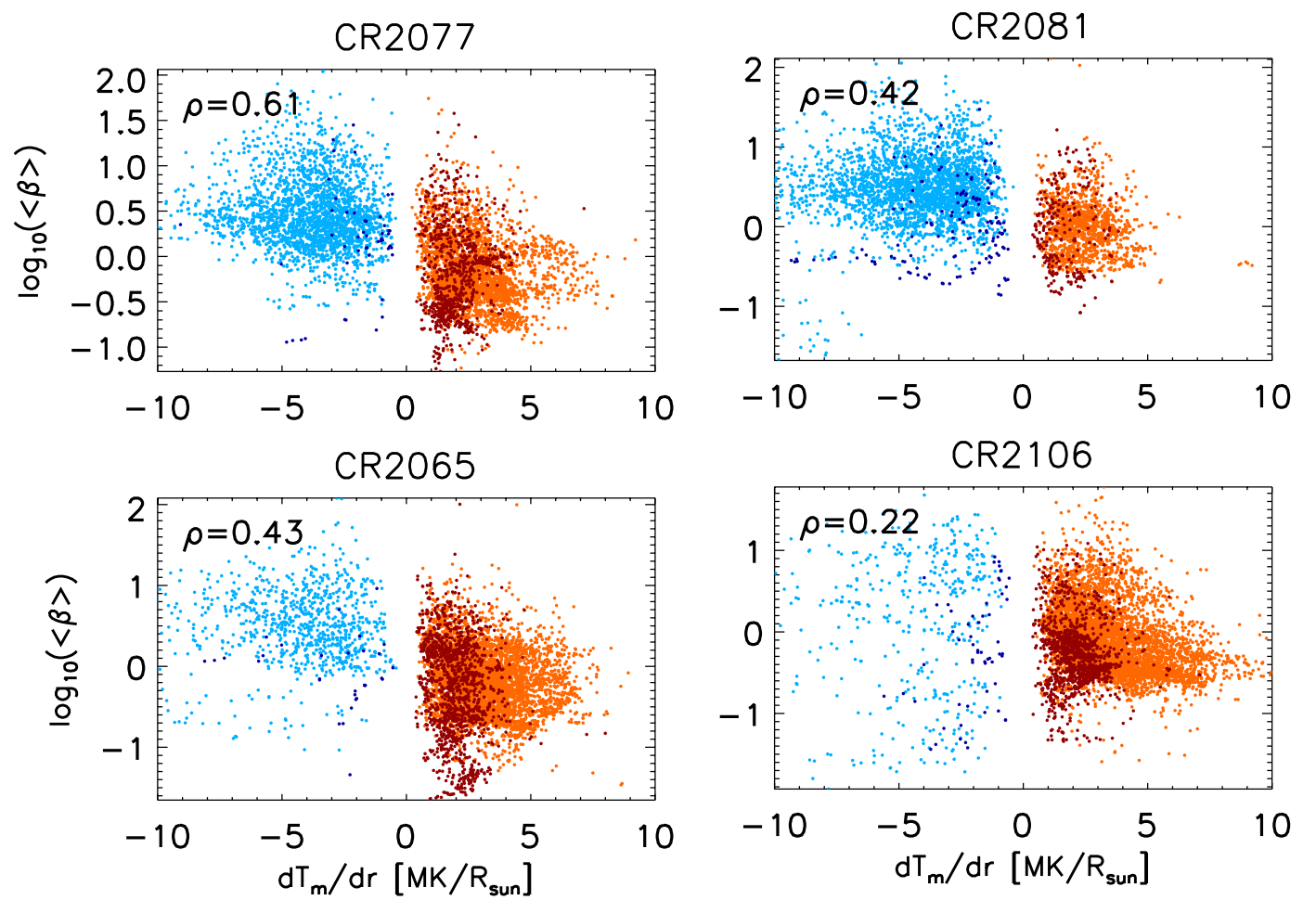

Figure 12. Scatter plots of the fitted temperature gradient $d T_{m} / d r$ vs. the mean value $\langle\beta\rangle$ (logarithmic scale), averaged over heights $1.035-1.20 R_{\odot}$ along each leg, for the same rotations shown in Figure 10, using the same color code to distinguish the different populations of loops.

(A color version of this figure is available in the online journal.)

Table 2

Median and Standard Deviation of the Distribution of the Mean Plasma Parameter $\langle\beta\rangle$, Averaged between Heights 1.035 and $1.20 R_{\odot}$ Along Each Leg, and the $\beta$ Value of Each Leg at $1.035 R_{\odot}$, Discriminating their Different Types: Up, Down, Small, and Large for the Same Rotations Shown in Figure 10

\begin{tabular}{llcccc}
\hline \hline CR & Leg Type & $\begin{array}{c}\text { Median } \\
\langle\beta\rangle\end{array}$ & $\begin{array}{c}\text { Standard Deviation } \\
\langle\beta\rangle\end{array}$ & $\begin{array}{c}\text { Median } \beta \text { at } \\
1.035 R_{\odot}\end{array}$ & $\begin{array}{c}\text { Standard Deviation } \beta \text { at } \\
1.035 R_{\odot}\end{array}$ \\
\hline 2065 & Small up & 0.64 & 1.89 & 0.41 & 1.23 \\
& Small down & 3.18 & 8.06 & 0.92 & 2.93 \\
& Large up & 0.61 & 3.26 & 0.28 & 0.88 \\
\hline \multirow{2}{*}{2077} & Large down & 1.16 & 1.78 & 0.71 & 1.76 \\
& Small up & 0.54 & 1.90 & 0.38 & 4.44 \\
& Small down & 2.63 & 6.98 & 1.06 & 1.78 \\
& Large up & 0.67 & 2.58 & 0.22 & 1.18 \\
\hline 2081 & Large down & 1.56 & 5.24 & 0.46 & 3.57 \\
& Small up & 0.94 & 4.76 & 0.51 & 1.43 \\
& Small down & 2.98 & 7.06 & 1.10 & 0.89 \\
\hline \multirow{2}{*}{2106} & Large up & 0.90 & 2.22 & 0.29 & 1.79 \\
& Large down & 0.90 & 3.60 & 0.57 & 3.37 \\
& Small up & 0.68 & 2.58 & 0.41 & 0.60 \\
& Small down & 2.34 & 6.07 & 0.88 & 1.76 \\
\hline
\end{tabular}

median $\langle\beta\rangle$ of large down loops to that of large up loops ranges between 1.2 and 2.3, except for CR-2081, which shows similar values of the median $\langle\beta\rangle$.

Figure 13 shows Carrington maps of the plasma $\beta$ at a height $1.075 R_{\odot}$, similar to those shown for CR-2068 in Figure 7 of Vásquez et al. (2011). The color scale and saturation at $\beta=5$ have been chosen so that the $\beta=1$ level is clearly demarcated by the green/red division. These maps can be visually compared to the up/down location maps in Figures 6 and 8, showing that the evolution of the high beta region is morphologically consistent with the up/down distribution. The vast majority of down loops is located in the evolving $\beta>1$ region (red, violet, and white), while the up loops are mostly located in the $\beta<1$ region (green and black).

The total number of up and down loops was computed for each of all $11 \mathrm{CRs}$. While the latitudinal distribution of up/down loops is similar in all cases, the most noticeable change from one rotation to another is the relative fraction of up and down loops. Figure 14 shows the evolution of the relative fractions of the total number of up loops (orange diamonds) and down loops (light blue diamonds) as a function of CR. As an indicator of the global solar activity level, we also overplot the Brussels 

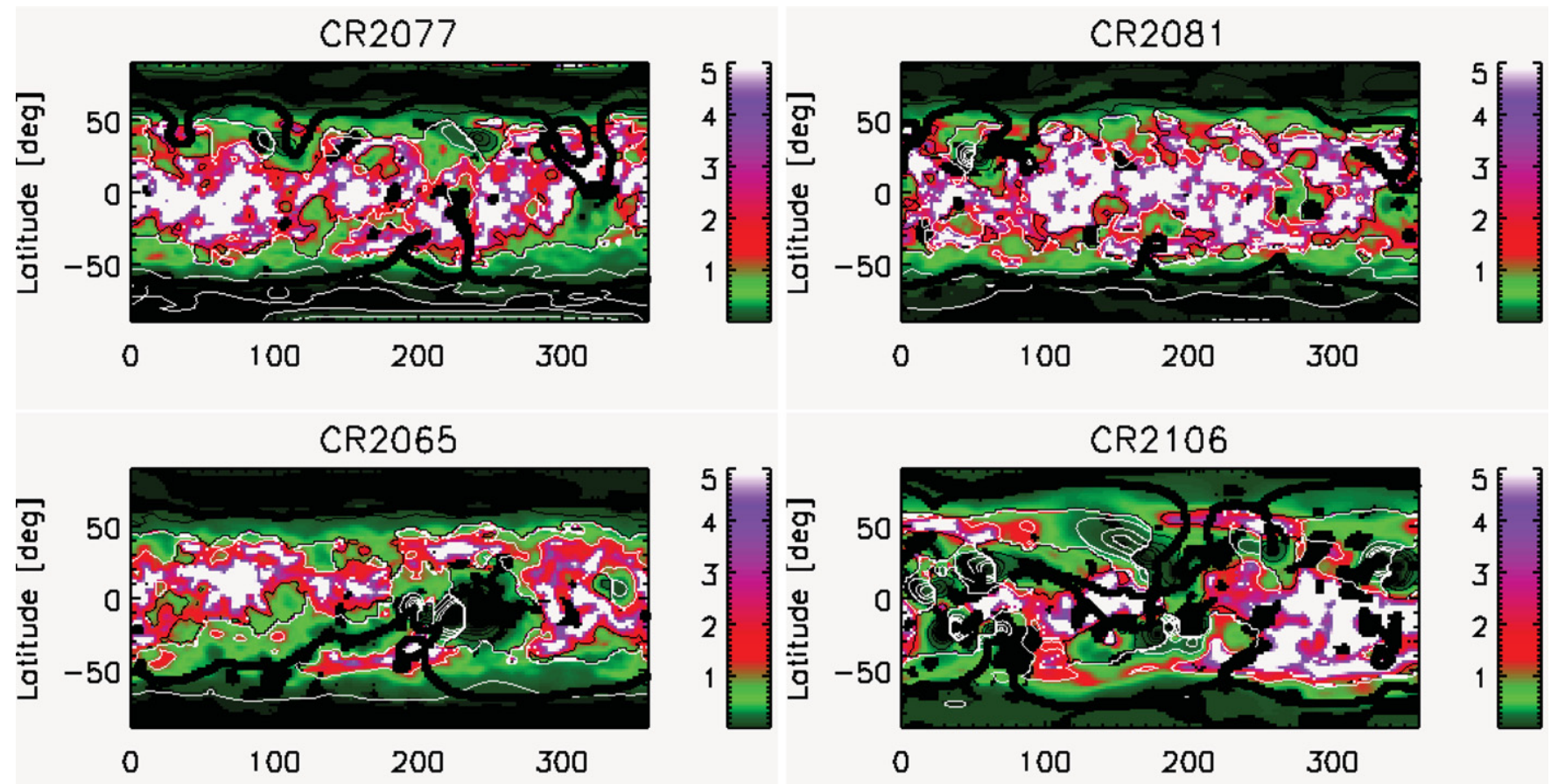

Figure 13. Carrington maps of the plasma $\beta$ at $1.075 R_{\odot}$ for the same four rotations selected in Figure 12 .

(A color version of this figure is available in the online journal.)

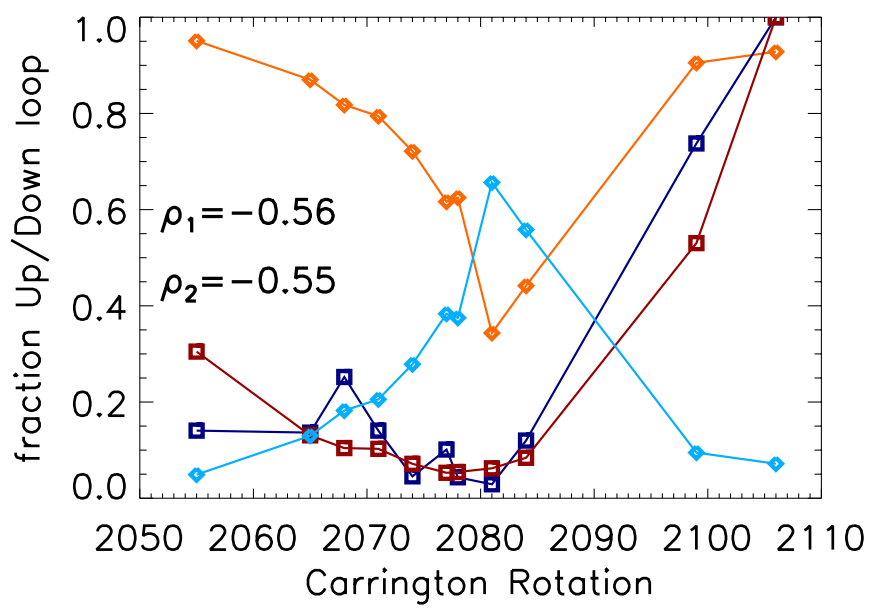

Figure 14. Progression of the up and down loop fractions, indicated as orange and light blue diamonds, respectively. Overplotted is the Brussels International Sunspot Number, both its monthly raw data (dark blue squares) and its monthly smoothed value (red squares). The sunspot number values have been divided by its maximum value over the analyzed period, so that the peak value " 1 " corresponds to 33.5 sunspots for the smoothed number and 32.1 sunspots from the raw data. The population of down loops maximizes at CR-2081. The linear Pearson correlation coefficient between the down loop fraction and the smoothed (raw) sunspot number is $\rho_{1}=-0.56(-0.55)$.

(A color version of this figure is available in the online journal.)

International Sunspot Number. The blue squares represent the monthly raw data and the red squares represent the monthly smoothed data. Figure 14 reveals the most important single result of this paper: the down loop population is maximized at the solar minimum and diminished away from it, while the opposite behavior is observed for up loops. Most noticeably, the peak of the down loop population occurred during the rotation that exhibited the absolute minimum in the Brussels sunspot number progression, namely, CR-2081, which was less than one sunspot during that rotation. This tendency has been quantified by computing the linear Pearson correlation coefficient between the down loop fraction and the monthly raw (smoothed) sunspot number, which results in $\rho_{1}=-0.55(-0.56)$.

\section{DISCUSSION AND CONCLUSIONS}

We performed a statistical study of the temperature structure of the closed field region of the global corona for $11 \mathrm{CRs}$ around the last minimum of solar activity. We carried on this study using MLDT, which traces the three-dimensional tomographic reconstructions of the coronal electron density and temperature along magnetic field lines of global PFSS models. We selected five rotations during the activity declining phase of SC-23 before the last solar minimum, three rotations during the deep minimum period, and three rotations during the rising phase of SC-24. For each rotation we analyzed the thermodynamic properties of the coronal loops by means of the MLDT approach.

We found that down loops are characteristic of the QS during the solar minimum. Our major finding is that the down loop fraction is maximized during deep minimum, specifically in CR-2081, coincident with the absolute minimum of the monthly sunspot number. The up loop fraction is minimal at deep minimum and its population increased away from minimum. Down loops are ubiquitous at low latitudes during deep minimum, with more than $91 \%$ of their population being located within the latitude range $\pm 30^{\circ}$. The up loops are located at middle latitudes, with more than $75 \%$ of their population being outside the latitude range $\pm 30^{\circ}$ for most rotations. The majority of down loops are located in quiet regions, while up loops are located in both quiet as well as in the few active regions (ARs) that existed during the time period farther from minimum. Additionally, we performed a DEM analysis based on images simultaneously taken by the three EUVI-B bands, verifying the presence of inward temperature gradients at low 
latitudes and outward ones at mid-latitudes, independently of the tomographic results. In this context, the fact that down loops are a characteristic of deep minimum at low latitudes, while they are much less present away from the minimum, supports the idea of heating mechanisms being modulated by the evolving coronal magnetic field.

In the active regions of the corona, the PFSS models used in this work may of course not be accurate; however, the conclusions drawn here are robust. In the case of down loops, the vast majority of them are located in the QS regions for all the rotations analyzed. Concerning up loops, active regions were very few and compact for the rotations analyzed, having a negligible impact on the results. Rotations CR-2055 and CR-2065 showed only two significantly large ARs each, ${ }^{7}$ and those had no impact on the results as virtually no up loops were selected from those regions due to the rejection criteria (Section 2.1). Rotation CR-2068 (see Vásquez et al. 2011) showed a more extended complex of three ARs, but very few up loops ended up being selected from those regions, so their impact on the results is very minor. For the deep minimum period, rotations CR-2071, CR-2074, CR-2077, CR-2078, CR2081, and CR-2084 showed no or one significantly large AR each, and again virtually no up loops were selected from those. The last two rotations analyzed in this work showed a greater number of significantly large ARs, 5 in the case of CR-2099 and 10 in the case of CR-2106. In both cases the great majority of the loops selected for analysis do not belong to the ARs.

Down loops are likely to be indicative of heating strongly concentrated in their footpoints, at the coronal base. At $1.035 R_{\odot}$ (our lowest observed height), we found that the median value of $\beta$ in the small (apex below $1.2 R_{\odot}$ ) down loops was $\approx 1$, while in small up loops it was significantly less than unity (cf. Table 2). At larger heights, this difference in $\beta$ between down and up loops is even greater, as indicated by the value $\langle\beta\rangle$ (averaged along each leg between heights 1.035 and $1.20 R_{\odot}$ ), which is 2-3 for small down loops, and significantly below unity for small up loops. For the CRs we analyzed, the median value of $\langle\beta\rangle$ for small down loops is 3-5 times larger than for small up loops. Differences between large (apex above $1.2 R_{\odot}$ ) down loops and large up loops are less significant, but the statistics of large loops is very poor. Values of $\beta \gtrsim 1$ in the core region of the quiescent streamer belt have been already found in previous observational works (Li et al. 1998; Kohl et al. 1995; Vásquez et al. 2011) as well as modeling efforts (Suess et al. 1996; Wang et al. 1998; Vásquez et al. 2003). The condition $\beta \sim 1$ is optimal for mode conversion between Alfvénic and compressive modes (e.g., slow and fast modes) that can then be damped. In recent 2.5 dimensional MHD simulations, covering the photosphere to the interplanetary medium, Matsumoto \& Suzuki (2012) found that shock heating by the dissipation of the slow mode wave plays a fundamental role in the heating of the corona. We speculate then that down loops with $\beta \gtrsim 1$ allow for efficient conversion of Alfvén waves to compressive modes, so that the damping is enhanced at low heights. In up loops, where $\beta$ is low, this heat source is not available, and the Alfvén waves propagate to larger heights, where wave reflection and turbulent cascades lead to a more uniform heating.

In a recent observational work based on Extreme Ultraviolet Imaging Spectrometer (EIS) data, Hahn et al. (2012) measured the variation of spectral UV line widths in the range of heights from 1.05 to $1.4 R_{\odot}$ over a polar coronal hole. They separated

\footnotetext{
www.solarmonitor.org
}

the non-thermal component of the line width, which is expected to be proportional to the Alfvén wave amplitude (Hassler et al. 1990; Banerjee et al. 1998). They found that, starting at heights as low as $1.1 R_{\odot}$, the non-thermal component decreases with height in a way that deviates from the predicted dependence for undamped waves (Moran 2001). Their observations indicate that Alfvén waves are being damped at surprisingly low heights in the polar coronal hole. The mechanism responsible for those observations may also be operating at low latitudes in the closed magnetic fields. In view of our results, future work will include the exploration of the presence of such evidence in EIS data scans of low-latitude regions during the last minimum.

There is extensive evidence of the ubiquitous presence of Alfvén waves in the Sun, starting at chromospheric levels (Jess et al. 2009) and the transition region (McIntosh et al. 2011) up to coronal heights (Tomczyk et al. 2007; Tomczyk \& McIntosh 2009; Jess et al. 2012) and the heliosphere (Belcher \& Davis 1971). A primary dissipation mechanism of Alfvén waves is that of collisions, with typical damping scale lengths $\gtrsim 1 R_{\odot}$ (Cranmer 2002). Alternative wave damping mechanisms can operate at much smaller scales, such as phase mixing, turbulent cascade, and resonant absorption in inhomogeneous plasmas (Heyvaerts \& Priest 1983; Matthaeus et al. 1999; Goossens et al. 2011; Dong \& Paty 2011). Observational evidence for wave damping at low heights has been recently found by Hahn et al. (2012). They studied optically thin spectral lines observed by the EIS on board Hinode in coronal holes. They measured the amplitude of the non-thermal line broadening of the lines, thought to be proportional to that of the Alfvén waves (Banerjee et al. 1998; Doyle et al. 1998; Moran 2001; Banerjee et al. 2009). They found that amplitude to exhibit downward gradients in height, between 1.1 and $1.3 R_{\odot}$, providing strong evidence of Alfvén wave damping occurring in that range of heights. Recent observational results based on Hinode (De Pontieu et al. 2007) indicate that the power carried by chromospheric Alfvén waves is even larger than thought previously, and that only a fraction of them would suffice to provide the energy requirements for the coronal heating and the acceleration of the solar wind.

Wave-driven global coronal models contain two key parameters that govern the behavior of the heating term, namely, the Alfvén wave dissipation length and its reflection coefficient. The dissipation length can be inferred from observations, such as those by Hahn et al. (2012). The Alfvén wave reflection coefficient is rather uncertain. In the context of a global coronal model, Z. Huang et al. (2013, in preparation) treat the reflection coefficient as a free parameter. They show that for large enough reflection coefficients near the Sun, a fully three-dimensional solar corona model can reproduce the up and down loops observed in the solar corona.

In view of our finding of down loops and their characteristic $\beta \gtrsim 1$ values, we propose the following physical picture for the QS heating. In the $\beta \gg 1$ photosphere, Alfvén waves are created from 3-5 minute oscillations and chromospheric shocks (Matsumoto \& Suzuki 2012). Along loops for which $\beta<1$, which we found to be up loops, Alfvén waves are damped by reflection and turbulent cascade, thus heating is not isolated in the bottoms of the loops. Along loops for which $\beta \gtrsim 1$, which we found to be down loops, the Alfvén waves can be efficiently converted into compressive modes, which are quickly damped at the transition region due to the high sound speed gradient and thermal conduction. This puts the majority of the heating 
at the coronal base. In this scenario, the heating of both up and down loops finds its energy source in exactly the same Alfvén waves with origins in the photosphere. Depending on the value of $\beta$ in the low corona, these waves are then dissipated through different mechanisms, but the total amount of Alfvén wave energy available is about the same, leading to similar temperatures in both types of loops, as observed in the MLDT results. This picture naturally explains why the QS plasma appears as diffuse emission, not allowing individual loops to be seen, as the proposed processes occur in a fairly homogeneous fashion. This speculative scenario will be further investigated through extended MLDT analysis and MHD modeling (Z. Huang et al. 2013, in preparation).

The authors acknowledge the review by the anonymous referee that helped to improve the clarity of the manuscript and enrich the discussion section. F.A.N. acknowledges the CONICET Type I pre-doctoral fellowship that supports his participation in this research.

\section{REFERENCES}

Aschwanden, M., \& Schrijver, C. J. 2002, ApJS, 142, 269

Banerjee, D., Pérez-Suárez, D., \& Doyle, J. G. 2009, A\&A, 501, L15

Banerjee, D., Teriaca, L., Doyle, J. G., \& Wilhelm, K. 1998, A\&A, 339, 208

Belcher, J. W., \& Davis, L. 1971, JGR, 76, 3534

Cranmer, S. R. 2002, SSRv, 101, 229

De Pontieu, B., McIntosh, S. W., Carlsson, M., et al. 2007, Sci, 318, 1574

Dong, C. F., \& Paty, C. S. 2011, PhP1, 18, 030702

Doyle, J. G., Banerjee, D., \& Perez, M. E. 1998, SoPh, 181, 91

Frazin, R. A., Vásquez, A. M., \& Kamalabadi, F. 2009, ApJ, 701, 547

Goossens, M., Erdélyi, R., \& Ruderman, M. S. 2011, SSRv, 158, 289

Hahn, M., Landi, E., \& Savin, D. W. 2012, ApJ, 753, 36
Hassler, D. M., Rottman, G. J., Shoub, E. C., \& Holzer, T. E. 1990, ApJL, 348, L77

Heyvaerts, J., \& Priest, E. R. 1983, A\&A, 117, 220

Huang, Z., Frazin, R. A., Landi, E., et al. 2012, ApJ, 755, 86

Jess, D. B., Mathioudakis, M., Erdélyi, R., et al. 2009, Sci, 323, 1582

Jess, D. B., Shelyag, S., Mathioudakis, M., et al. 2012, ApJ, 746, 183

Kohl, J. L., Esser, R., Gardner, L. D., et al. 1995, SoPh, 162, 313

Li, J., Raymond, J. C., Acton, L. W., et al. 1998, ApJ, 506, 431

Matsumoto, T., \& Suzuki, T. K. 2012, ApJ, 749, 8

Matthaeus, W. H., Zank, G. P., Oughton, S., Mullan, D. J., \& Dmitruk, P. 1999, ApJ, 523, 93

McIntosh, S. W., de Pontieu, B., Carlsson, M., et al. 2011, Natur, 475,477

Moran, T. G. 2001, A\&A, 374, L9

Nuevo, F. A., Vásquez, A. M., Frazin, R. A., Huang, Z., \& Manchester, W. B., IV 2012, in Proc. IAU Symp. 286, Comparative Magnetic Minima: Characterizing Quiet Times in the Sun and Stars, ed. C. H. Mandrini \& D. F. Webb (Cambridge: Cambridge Univ. Press), 238

Prasad, S. K., Singh, J., \& Ichimoto, K. 2013, ApJL, 765, L46

Riley, P., Linker, J. A., Mikić, Z., et al. 2006, ApJ, 653, 1510

Serio, S., Peres, G., Vaiana, G. S., Golub, L., \& Rosner, R. 1981, ApJ, 243, 288

Shearer, P., Frazin, R. A., Hero, A. O., III, \& Gilbert, A. C. 2012, ApJL, 749, L8

Suess, S. T., Wang, A. H., \& Wu, S. T. 1996, JGR, 101, 19957

Tomczyk, S., \& McIntosh, S. W. 2009, ApJ, 697, 1384

Tomczyk, S., McIntosh, S. W., Keil, S. L., et al. 2007, Sci, 317, 1192

Tóth, G., van der Holst, B., \& Huang, Z. 2011, ApJ, 732, 102

Vásquez, A. M., Frazin, R. A., Huang, Z., Manchester, W. B., IV, \& Shearer, P. 2012, in Proc. IAU Symp. 286, Comparative Magnetic Minima: Characterizing Quiet Times in the Sun and Stars, ed. C. H. Mandrini \& D. F. Webb (Cambridge: Cambridge Univ. Press), 123

Vásquez, A. M., Frazin, R. A., \& Manchester, W. B., IV 2010, ApJ, 715, 1352

Vásquez, A. M., Huang, Z., Manchester, W. B., IV, \& Frazin, R. A. 2011, SoPh, 274,259

Vásquez, A. M., van Ballegooijen, A. A., \& Raymond, J. C. 2003, ApJ, 598, 1361

Wang, Y. M., Sheeley, N. R., Jr., Walters, J. H., et al. 1998, ApJL, 498, L165 